\title{
Kernels of Linear Representations of Lie Groups, Locally Compact Groups, and Pro-Lie Groups
}

\author{
Markus Stroppel
}

\begin{abstract}
For a topological group $G$ the intersection $\operatorname{KOR}(G)$ of all kernels of ordinary representations is studied. We show that $\operatorname{KOR}(G)$ is contained in the center of $G$ if $G$ is a connected pro-Lie group. The class $\operatorname{KOR}(\mathcal{C})$ is determined explicitly if $\mathcal{C}$ is the class CONNLIE of connected Lie groups or the class ALMCONNLIE of almost connected Lie groups: in both cases, it consists of all compactly generated abelian Lie groups. Every compact abelian group and every connected abelian pro-Lie group occurs as $\operatorname{KOR}(G)$ for some connected pro-Lie group $G$. However, the dimension of $\operatorname{KOR}(G)$ is bounded by the cardinality of the continuum if $G$ is locally compact and connected. Examples are given to show that $\operatorname{KOR}(\mathcal{C})$ becomes complicated if $\mathcal{C}$ contains groups with infinitely many connected components.
\end{abstract}

\section{The questions we consider and the answers that we have found}

In the present paper we study (Hausdorff) topological groups. If all else fails, we endow a group with the discrete topology.

For any group $G$ one tries, traditionally, to understand the group by means of representations as groups of matrices. To this end, one studies the continuous homomorphisms from $G$ to $\mathrm{GL}_{n} \mathbb{C}$ for suitable positive integers $n$; so-called ordinary representations. This approach works perfectly for finite groups because any such group has a faithful ordinary representation but we may face difficulties for infinite groups; there do exist groups admitting no ordinary representations apart from the trivial (constant) one. See 10.8 below.

The possible images of $G$ under ordinary representations are called linear groups over $\mathbb{C}$. More generally, one may study linear groups over arbitrary fields. See [47] and [50] for overviews of results in that direction. We just note here that for every free abelian group $A$ there exists at least one field $F$ such that $A$ is a linear group over $F$. However, there do exist abelian groups that are not linear over any field, see [33], cf. [47. 2.2]. Note also that quotients of linear groups may fail to be linear, cf. [47, Ch. 6]. This phenomenon will play a role in 4.1 below.

In the present notes, we are mainly interested in that part of $G$ that cannot be understood by means of ordinary representations, namely, the intersection $\operatorname{KOR}(G)$ of all 
kernels of ordinary linear representations. A detailed overview over the results of the present paper will be given in 1.5 below; we give some coarse indications here before we introduce more specific notation.

We will show that $\operatorname{KOR}(G)$ is a central subgroup of $G$ if $G$ belongs to the class CONNPROLIE of all connected pro-Lie groups (in particular, if $G$ is locally compact and connected). Moreover, we investigate the class $\operatorname{Kor}(\mathcal{G}):=\{\operatorname{KoR}(G) \mid G \in \mathcal{G}\}$ for different classes $\mathcal{G}$ of groups. For the class CONNLIE of connected Lie groups, in particular, we show in 4.5 that KOR(CONNLIE) is the class CGAL of compactly generated abelian Lie groups. Thus this class is as large as possible (after the observation that $\operatorname{KOR}(G)$ is central for each $G \in$ CONNLIE, cf. 3.4). The class $\operatorname{KOR}($ CONNPROLIE) contains all connected abelian pro-Lie groups and all compact abelian groups, see 6.3. For the class CONNLCG of connected locally compact groups it turns out that there is a somewhat surprising bound on the dimension of members of $\operatorname{KOR}(\mathrm{CONNLCG})$, see 7.15

1.1 Notation. For topological groups $G, H$ let $\operatorname{Hom}(G, H)$ denote the set of continuous homomorphisms from $G$ to $H$. If $G$ and $H$ are (topological) vector spaces over $\mathbb{F}$, we write $\operatorname{Hom}_{\mathbb{F}}(G, H)$ for the set of continuous $\mathbb{F}$-linear homomorphisms. We put

$$
\operatorname{OR}(G):=\bigcup_{n \in \mathbb{N}} \operatorname{Hom}\left(G, G_{n} \mathbb{C}\right) \text {, then } \operatorname{KoR}(G)=\bigcap_{\rho \in \operatorname{OR}(G)} \operatorname{ker} \rho .
$$

Clearly $\operatorname{KOR}(G)$ is a closed normal subgroup of $G$, in fact, it is fully invariant (i.e., each endomorphism of the topological group $G$ maps $\operatorname{KOR}(G)$ into itself, see 2.1 below).

In order to keep notation simple, we also consider continuous homomorphisms from $G$ to the group GL( $V)$ of all linear bijections of a vector space $V$ of finite dimension $n$ over $\mathbb{F} \in\{\mathbb{R}, \mathbb{C}\}$. Note that this does not mean that we consider our problem in greater generality because $\mathrm{GL}(V)$ is isomorphic to $\mathrm{GL}_{n} \mathbb{F} \leq \mathrm{GL}_{n} \mathbb{C}$.

1.2 Remarks. Our present problem bears some similarity to questions that arise in character theory. E.g., for a locally compact abelian group $G$ the ordinary representations may be reduced to collections of homomorphisms from $G$ into $G L_{1} \mathbb{C} \cong \mathbb{C}^{\times} \cong \mathbb{R} \times \mathbb{T}$ where $\mathbb{T}:=\mathbb{R} / \mathbb{Z}$ as usual. The elements of $G^{*}:=\operatorname{Hom}(G, \mathbb{T})$ are called characters of $G$ while those of $\operatorname{Hom}(G, \mathbb{R})$ are the real characters 1 . Since $\operatorname{Hom}(\mathbb{R}, \mathbb{T})$ separates points we have $\bigcap_{\chi \in G^{*}} \operatorname{ker} \chi \subseteq \bigcap_{\rho \in \operatorname{Hom}(G, \mathbb{R})} \operatorname{ker} \rho$. Pontryagin's duality theory for locally compact abelian groups (cf. [45, Ch. F]) uses characters, it rests on the fact that $\operatorname{Hom}(G, \mathbb{T})$ separates points for each locally compact abelian group. This is no longer true for arbitrary topological abelian groups, cf. [15, 23.32]. The intersection over the kernels of real characters has been identified in [9], cf. [48], [3].

\footnotetext{
${ }^{1}$ One should not confuse this notion of real character (of topological abelian groups) with the usage of the term in the theory of characters of finite groups where it denotes a class function assuming real values, cf. [8. p. $56 \mathrm{ff}$ ]. Take the direct product $\prod_{p \in \mathbb{P}} \mathbb{Z}\left(p^{\infty}\right)$ with the discrete topology. The direct sum $\bigoplus_{p \in \mathbb{P}} \mathbb{Z}\left(p^{\infty}\right) \cong \mathbb{Q} / \mathbb{Z}$ is the torsion subgroup, but the full product is isomorphic to $\mathbb{Q}^{\left(2^{\aleph_{0}}\right)} \oplus \mathbb{Q} / \mathbb{Z}$. The latter is isomorphic to $(\mathbb{R} / \mathbb{Z})_{\text {discr }}$ and to $(\mathbb{R} \times \mathbb{Q} / \mathbb{Z})_{\text {discr. }}$. The projection onto the torsionfree summand $\mathbb{Q}^{\left(2^{\aleph_{0}}\right)} \cong \mathbb{R}_{\text {discr }}$ is a real character, indeed.
} 
1.3 Remark. Our present problem disappears if we take a local (or, rather, an infinitesimal) point of view. Indeed Ado's Theorem ([1], see [2] for an English translation, cf. also [12], [27], [46]) asserts that every Lie algebra $\mathfrak{g}$ of finite dimension over $\mathbb{R}$ or $\mathbb{C}$ has a faithful ordinary representation; i.e. a faithful homomorphism into $\mathfrak{g l}_{n} \mathbb{R}$ for some $n$. Thus a single, suitably chosen representation suffices to show that $\operatorname{Ror}(\mathfrak{g})$ is trivial, where

$$
\mathfrak{H o r}(\mathfrak{g}):=\bigcap_{n \in \mathbb{N}} \bigcap_{\rho \in \operatorname{Hom}\left(\mathfrak{g}, \mathfrak{g l}_{n} \mathrm{C}\right)} \operatorname{ker} \rho .
$$

For a pro-Lie algebra $g$ (i.e., a projective limit $g$ of Lie algebras of finite dimension over $\mathbb{R}$ such that $\mathrm{g}$ is complete as a topological vector space, cf. [23, Ch. 7]) one also knows that $\operatorname{Ror}(\mathfrak{g})$ is trivial.

In particular, there is no useful relationship between $\operatorname{KoR}(G)$ and $\operatorname{Ror}(\mathfrak{L}(G))$ if $G$ is a group which has Lie algebra $\mathfrak{L}(G)$ (in the sense of 3.1]below). However, the Lie algebra will be useful to construct ordinary representations of a pro-Lie group, see 3.4 .

1.4 Some classes of groups. The following will be of interest to us here:

TG : topological Hausdorff groups,

PROLIE : pro-Lie groups, i.e., complete projective limits of Lie groups,

CG : compact groups,

LCG : locally compact groups,

LCA : locally compact abelian groups,

LIE : Lie groups (without separability assumptions, i.e., including all discrete groups),

SEPLIE : separable Lie groups (i.e., the $\sigma$-compact members of LIE),

CGAL $=$ LCA $\cap$ SEPLIE : compactly generated abelian Lie groups,

and the classes $A B \mathcal{G}, \operatorname{CONNG}$ or ALMCONNG consisting of the abelian, connected or almost connected members of the class $\mathcal{G}$, respectively. Here a group $G$ is called almost connected if the quotient $G / G_{0}$ modulo the connected component $G_{0}$ is compact.

For sentimental historical reasons, we write LCA and CA instead of the more systematic $A B L C G$ and $A B C G$, respectively. The diagram in Figure11indicates the inclusions between these classes.

Note that we only consider Hausdorff groups; otherwise, the closure of the trivial subgroup would occur inside $\operatorname{KOR}(G)$ throughout.

As it is customary in the theory of locally compact groups, we do not include separability in the definition of a Lie group. This means that every discrete group is a Lie group, and it secures (via the solution of Hilbert's Fifth Problem, cf. [49], [34]) that every locally euclidean group is a Lie group. Thus the additive group $\mathbb{R}_{\text {discr }}$ of real numbers with the discrete topology belongs to LIE $\backslash$ SEPLIE (it is not a member of CGAL), and the identity from $\mathbb{R}_{\text {discr }}$ to $\mathbb{R}$ is a bijective morphism of Lie groups which is not open. If one wants to use the Open Mapping Theorem (which is indeed one of the major reasons to require separability) one has to be careful and make sure that the domain of the mapping is $\sigma$-compact. Note that every closed subgroup of an almost connected Lie group belongs to SEPLIE. In our present context separability does not appear to be of much help (see 3.7(a), 8.1) while almost connectedness is a useful condition for Lie groups (cf. (4.8) where it actually means that the number of connected components is finite. 


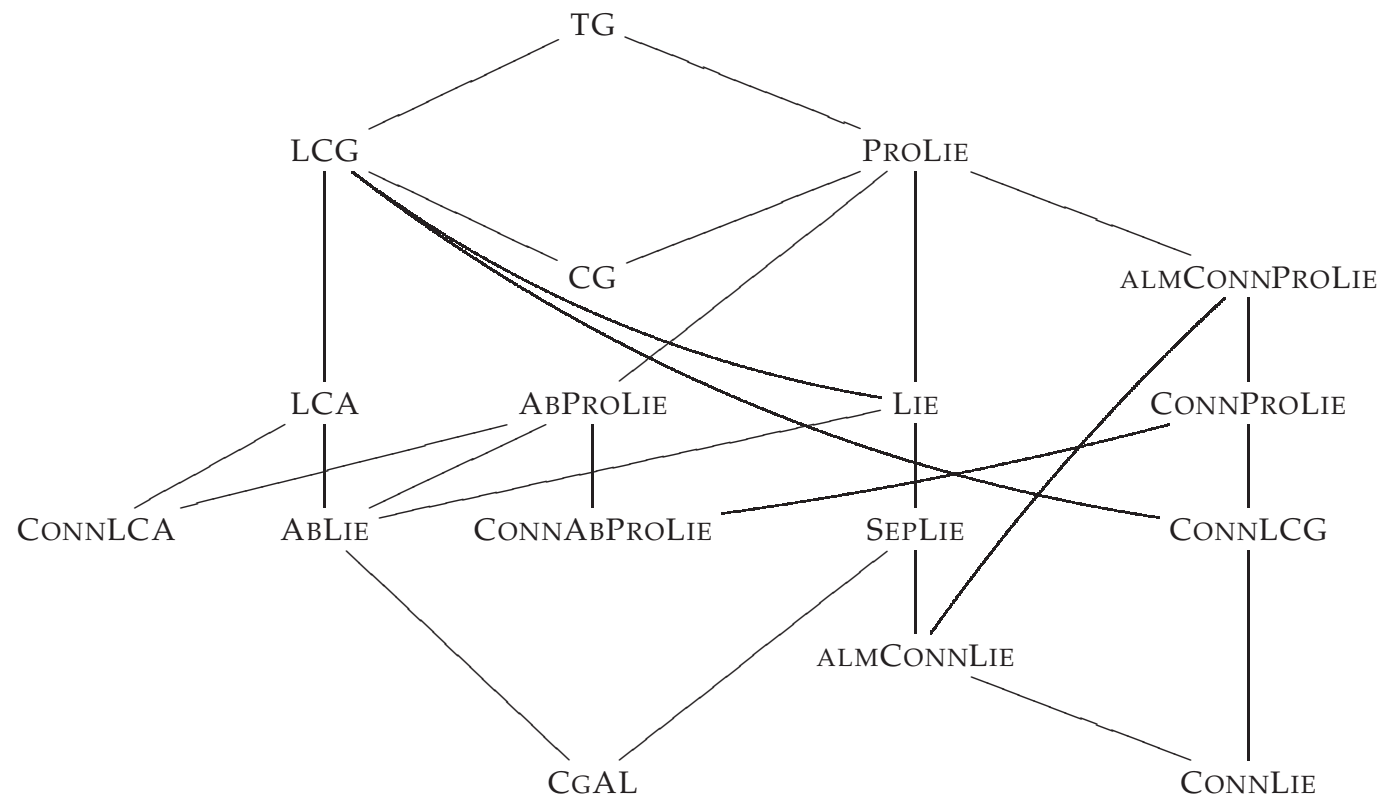

Figure 1: Inclusions between classes of topological groups

1.5 Overview of results. In the present paper, we obtain the following.

- KOR is a functor that preserves products, see 2.1, 2.7

- For each $G \in$ ConNProLie we have $\operatorname{Kor}(G) \subseteq Z(G) \cap \overline{G^{\prime}}$, see 3.4 and 3.8

- $\operatorname{Kor}($ almConnlie $)=\operatorname{Kor}($ CONnLie $)=$ CGAL, see 4.5 and 4.8.

- $\operatorname{Kor}(G)$ is trivial for every compact and every abelian proto-Lie group $G$, see 5.1 .

- For $G \in$ CONNLCG the connected component $(\operatorname{KOR}(G))_{0}$ of $\operatorname{KOR}(G)$ has a finitely generated dense subgroup. Thus the weight of $(\operatorname{KOR}(G))_{0}$ is bounded by $2^{\aleph_{0}}$, see 7.14

- If $G \in$ CONNLCG is solvable then $\overline{G^{\prime}}$ has a finitely generated dense subgroup, cf.7.12. This implies that the weight of $\operatorname{KOR}(G)$ is bounded by $2^{\aleph_{0}}$.

- The inclusions ConnAbProLie $\subset \Pi($ CGAL $\cup \mathrm{CA}) \subseteq \mathrm{KOR}($ CONNProLie $) \subseteq$ $\overline{\mathbf{S}}$ (CONNABPROLIE) are established in 6.3 and 9.2

- KOR(CONNLCG) contains those $A \in \mathrm{LCA}$ that possess a finitely generated dense subgroup, see 7.15 .

- For each $G \in$ CONNLCG there exist $A \in \mathrm{CA}$ and natural numbers $e, f$ such that the connected component $A_{0}$ is monothetic and $\operatorname{KOR}(G) \cong \mathbb{Z}^{f} \times A \times \mathbb{R}^{e}$, see7.15. In particular, the dimension of members of $\operatorname{KOR}(\mathrm{CONNLCG})$ is bounded by $2^{\aleph_{0}}$.

The latter two of these results mean that $\operatorname{KOR}(\mathrm{CONNLCG})$ is sandwiched between the class SMALLLCA of groups of the form $\mathbb{Z}^{f} \times F \times \mathbb{R}^{e}$ where $F$ is a compact group with a finitely generated dense subgroup and the class of groups of the form $\mathbb{Z}^{f} \times A \times \mathbb{R}^{e}$ where $A$ is compact and its connected component $A_{0}$ has a finitely generated dense subgroup; here $f$ and $e$ may be arbitrary natural numbers.

The classes KOR(SEPLIE) and KOR(LCG) are large and complicated, see 8.1, 8.2 
and 8.4. Some open problems are stated in Section 9. Relevant results about (non-) linear(ity of) groups are collected in Section 10 .

\section{Basic results}

In this section we discuss functoriality of KOR and the behavior of this functor with respect to homomorphisms (in particular quotients) and products.

2.1 Lemma. For $\varphi \in \operatorname{Hom}(G, H)$ we have $\varphi(\operatorname{KOR}(G)) \leq \operatorname{KoR}(H)$.

In particular, mapping $G$ to $\operatorname{KOR}(G)$ and $\varphi$ to $\operatorname{KOR}(\varphi):=\left.\varphi\right|_{\operatorname{KOR}(G)} ^{\operatorname{KOR}(H)}$ implements a functor from the category of topological groups to itself.

Proof. For each $\rho \in \mathrm{OR}(H)$ we have $\rho \circ \varphi \in \mathrm{OR}(G)$, and $\rho(\varphi(x))=$ id follows for each $x \in \operatorname{KOR}(G)$.

2.2 Proposition. Let $G$ be a group, and let $N$ be a subgroup of $G$.

(a) In any case the group $\operatorname{KOR}(N)$ is contained in $\operatorname{KOR}(G)$.

(b) If $N$ is a normal subgroup of $G$ then $\operatorname{KOR}(G) N / N$ is contained in $\operatorname{KOR}(G / N)$.

(c) If $N$ is normal and contained in $\operatorname{KOR}(G)$ then $\operatorname{KOR}(G) / N=\operatorname{KOR}(G / N)$.

(d) The subgroup $\operatorname{KOR}(G)$ is a radical in the sense that $\operatorname{KOR}(G / \operatorname{KOR}(G))$ is trivial.

Proof. The first two assertions follow from 2.1 using the inclusion map $\iota: N \rightarrow G$ and the canonical quotient map $q_{N} \in \operatorname{Hom}(G, G / N)$.

Now assume that $N$ is normal in $G$ and contained in $\operatorname{KOR}(G)$. For $x \in G \backslash \operatorname{KOR}(G)$ there exists $\rho \in \operatorname{OR}(G)$ with $\rho(x) \neq \mathrm{id}$, and $\rho$ factors as $\rho=\lambda \circ q_{N}$ because $N \leq$ $\operatorname{KOR}(G) \leq \operatorname{ker} \rho$. Thus there exists $\lambda \in \operatorname{OR}(G / N)$ with $\lambda(x N) \neq$ id. This means $\operatorname{KOR}(G) / N \geq \operatorname{KOR}(G / N)$; the inclusion $\operatorname{KOR}(G) / N \leq \operatorname{KOR}(G / N)$ is clear already. Thus (c) is established, and the last assertion follows.

From 2.2 (d) we see that $\operatorname{KOR}(G)$ is a sort of "radical" of the group $G$. Note that $\operatorname{Kor}(G / N)$ may be much larger than $\operatorname{KOR}(G) N / N$ if $N$ is not contained in $\operatorname{KoR}(G)$; the example in 4.1 is instructive here, again.

The category TG and its full subcategories ProLie, ConNProLie, ABProLie and CONNABPROLIE are closed under arbitrary products, and these are as expected (i.e., cartesian products with the product topology). A category of topological groups may contain products (in the categorical sense) that are endowed with a topology that is different from the product topology; e.g., this happens in the categories LCG and LCA (cf. [45, 16.22]). However, products in CONNLCG are the same as those in TG (in particular, they exist only if all but a finite number of the factors are compact), see [45, 16.23].

2.3 Definitions. For $\mathcal{G} \subseteq$ TG let $\mathbf{P}(\mathcal{G})$ denote the class of all cartesian products of finitely many members of $\mathcal{G}$. By $\Pi(\mathcal{G})$ we mean the class of arbitrary cartesian products of members of $\mathcal{G}$. In any case, we use the product topology. 
The class $\mathbf{S}(\mathcal{G})$ consists of all subgroups of members of $\mathcal{G}$ while $\overline{\mathbf{S}}(\mathcal{G})$ contains only the closed subgroups (which is more reasonable if one studies classes of complete groups as we do here).

By $\mathbf{Q}(\mathcal{G})$ we denote the class of all Hausdorff quotient groups of members of $\mathcal{G}$, i.e. the class of all groups $G / N$ where $G \in \mathcal{G}$ and $N$ is a closed normal subgroup of $G$. Finally, let $\widehat{\mathbf{Q}}(\mathcal{G})$ be the class of all (Hausdorff) completions of members of $\mathcal{G}$.

2.4 Remarks. A topological group need not have a completion, cf. [6, III $\S 3 \cdot 4$, Thm. 1]. The classes PROLIE and CONNPROLIE are not closed under $\mathbf{Q}$, see [23, 4.11]. However, Hausdorff quotients of pro-Lie groups are proto-Lie groups (see [23, 4.1]), i.e., they possess completions which belong to ProLIE, again. Thus $\widehat{\mathbf{Q}}($ ProLIE $)=$ ProLIE $\varsubsetneqq$ $\mathbf{Q}$ (PROLIE). Locally compact groups are complete anyway (see [6, III §3·3, Cor. 1], cf. [23, 1.31] or [45, 8.25]), and $\mathbf{Q}(\mathrm{LCG})=\mathrm{LCG}=\widehat{\mathbf{Q}}(\mathrm{LCG})$.

The following corollary to 2.2 is applicable to the class CONNLCG and its subclass CONNLIE, cf. 3.4. The class CONNPROLIE is problematic because it is not closed un$\operatorname{der} \mathbf{Q}$. Some restriction of the sort " $\operatorname{KOR}(G) \leq Z(G)$ " is necessary, cf. 8.4.

2.5 Corollary. Consider $\mathcal{G} \subseteq \mathrm{TG}$ with $\mathbf{Q}(\mathcal{G})=\mathcal{G}$ and assume that $\operatorname{KOR}(G) \leq \mathrm{Z}(G)$ holds for each $G \in \mathcal{G}$. Then $\operatorname{KOR}(\mathcal{G})=\mathbf{Q}(\operatorname{KOR}(\mathcal{G}))$.

2.6 Lemma ([40, 11.18, 13.13], cf. [23, 4.28]). Let $N$ be a normal subgroup of a pro-Lie group $G$. If $N$ is locally compact then $G / N$ is complete, and thus a pro-Lie group.

2.7 Proposition. The functor KOR preserves products in the category TG, in fact, we have $\operatorname{KoR}\left(\prod_{j \in J} A_{j}\right)=\prod_{j \in J} \operatorname{Kor}\left(A_{j}\right)$. For $\mathcal{G} \subseteq \mathrm{TG}$ this means $\mathbf{P}(\operatorname{KOR}(\mathcal{G}))=\operatorname{KoR}(\mathcal{G})$ if $\mathbf{P}(\mathcal{G})=\mathcal{G}$ and $\Pi(\operatorname{KoR}(\mathcal{G}))=\operatorname{KoR}(\mathcal{G})$ if $\Pi(\mathcal{G})=\mathcal{G}$.

Proof. Let $\prod_{j \in J} A_{j}$ be a cartesian product; the indexing set $J$ may be infinite. For $m \in J$ let $\eta_{m}: A_{m} \rightarrow \prod_{j \in J} A_{j}$ be the natural inclusion, and let $\pi_{m}: \prod_{j \in J} A_{j} \rightarrow A_{m}$ be the natural projection.

If $\rho: \prod_{j \in J} A_{j} \rightarrow \mathrm{GL}_{n} \mathbb{C}$ is an ordinary representation of the product then $\rho \circ \eta_{m}$ is an ordinary representation of $A_{m}$, and we obtain that the subgroup generated by $\bigcup_{j \in J} \eta_{j}\left(\operatorname{KoR}\left(A_{j}\right)\right)$ is contained in $\operatorname{KoR}\left(\prod_{j \in J} A_{j}\right)$. The product $\prod_{j \in J} \operatorname{KOR}\left(A_{j}\right)$ is the closure of that subgroup and thus also contained in $\operatorname{KOR}\left(\prod_{j \in J} A_{j}\right)$.

Conversely, consider $x \in \prod_{j \in J} A_{j} \backslash \prod_{j \in J} \operatorname{KoR}\left(A_{j}\right)$. Then there exists $m \in J$ such that $\pi_{m}(x) \notin \operatorname{KOR}\left(A_{m}\right)$ and we find an ordinary representation $\rho_{m}$ of $A_{m}$ with id $\neq$ $\rho_{m}\left(\pi_{m}(x)\right)=\left(\rho_{m} \circ \pi_{m}\right)(x)$. Since $\rho_{m} \circ \pi_{m}$ is an ordinary representation of $\prod_{j \in J} A_{j}$ this shows $x \notin \prod_{j \in J} A_{j} \backslash \operatorname{KOR}\left(\prod_{j \in J} A_{j}\right)$.

2.8 Lemma. Let $G$ be a solvable connected (not necessarily closed) subgroup of $\mathrm{GL}_{n} \mathbb{C}$. Then the following holds.

(a) There exists a sequence $V_{0}, \ldots, V_{n}$ of G-invariant subspaces such that for all $j<n$ we have $V_{j} \leq V_{j+1}$ and $\operatorname{dim} V_{j}=j$.

(b) For any sequence as in (a) the commutator group $G^{\prime}$ acts trivially on each $V_{j+1} / V_{j}$.

(c) There are no compact (in particular, no finite) subgroups in $G^{\prime}$ except the trivial one. 
Proof. Replacing $G$ by its closure in $\mathrm{GL}_{n} \mathbb{C}$ we lose neither solvability nor connectedness, cf. [45, 2.9, 7.5]. For closed connected solvable subgroups of $\mathrm{GL}_{n} \mathbb{C}$ assertion (a) is Lie's Theorem, cf. [14, Thm. 2.2, Ch. III].

Since $G$ acts as a subgroup of the abelian group $\mathrm{GL}\left(V_{j+1} / V_{j}\right) \cong \mathrm{GL}_{1} \mathbb{C} \cong \mathbb{C}^{\times}$on $V_{j+1} / V_{j}$ the commutator group $G^{\prime}$ acts trivially on that quotient.

Finally, let $C$ be a compact subgroup of $G^{\prime}$. We proceed by induction to show that $C$ acts trivially on $V_{j}$ for each $j \leq n$. Indeed, if $C$ acts trivially on $V_{j}$ it acts on $V_{j+1}$ as a subgroup of the group $N_{j}$ consisting of all $a \in \mathrm{GL}\left(V_{j+1}\right)$ acting trivially both on $V_{j}$ and on $V_{j+1} / V_{j}$. Now $N_{j}$ is isomorphic to the additive group $\operatorname{Hom}_{\mathbb{C}}\left(V_{j+1} / V_{j}, V_{j}\right)$. This is the additive group of a vector space of finite dimension over $\mathbb{R}$, and does not contain compact subgroups apart from the trivial one.

2.9 Corollary. Let $G$ be a solvable connected group. Then every compact subgroup of $G^{\prime}$ is contained in $\operatorname{KOR}(G)$.

Connectedness is a crucial assumption in 2.9, as finite groups show. Applications of 2.9 are given in 4.1 and 5.6 below. See also 10.1 (c) and 10.2

\section{Lie algebras and pro-Lie groups}

For a Lie group $L$ one model for the Lie algebra is the space $\operatorname{Hom}(\mathbb{R}, L)$ of all oneparameter subgroups, cf. [17]. This point of view works for quite general classes of topological groups, see [23, Ch. 2].

3.1 Definitions. For a topological group $G$ let $\mathfrak{L}(G)$ denote the space $\operatorname{Hom}(\mathbb{R}, G)$ endowed with the compact-open topology (i.e., the topology of uniform convergence on compact sets). We call exp: $\mathfrak{L}(G) \rightarrow G: X \mapsto X(1)$ the exponential map for $G$. Multiplication of $X \in \mathfrak{L}(G)$ by a scalar $r \in \mathbb{R}$ is given as $r X(t):=X(t r)$.

Addition and the Lie bracket are more involved, and not defined for arbitrary topological groups. We say that $G$ has a Lie algebra if the following conditions are satisfied:

(a) For all $X, Y \in \mathfrak{L}(G)$ there are elements $X+Y$ and $[X, Y]$ in $\mathfrak{L}(G)$ such that

$$
\begin{aligned}
(X+Y)(t) & =\lim _{n \rightarrow \infty}\left(X\left(\frac{t}{n}\right) Y\left(\frac{t}{n}\right)\right)^{n} \text { and } \\
{[X, Y]\left(t^{2}\right) } & =\lim _{n \rightarrow \infty} \operatorname{comm}\left(X\left(\frac{t}{n}\right), Y\left(\frac{t}{n}\right)\right)^{n^{2}}
\end{aligned}
$$

hold for all $t \in \mathbb{R}$; here $\operatorname{comm}(g, h):=g h g^{-1} h^{-1}$ is the group commutator.

(b) The set $\mathfrak{L}(G)$ is a topological Lie algebra with respect to these operations.

We say that $G$ has a generating Lie algebra if it has a Lie algebra and the range of the exponential map generates a dense subgroup of the connected component $G_{0}$.

3.2 Examples. In [23, 3.5] it is shown that every projective limit $G$ of Lie groups has a Lie algebra and, moreover, this Lie algebra $\mathfrak{L}(G)$ is a pro-Lie algebra: i.e., the filter basis of closed ideals of finite co-dimension in $\mathfrak{L}(G)$ converges to 0 and $\mathfrak{L}(G)$ is complete as a topological vector space. In particular, this Lie algebra is residually finite-dimensional; the homomorphisms to finite-dimensional Lie algebras separate the points.

Every almost connected pro-Lie group, and thus every almost connected locally compact group, has a generating Lie algebra, cf. [23, 4.22]. 
Our technical machinery culminates in the adjoint representation:

3.3 Lemma ([23, 2.27, 2.28, 2.30, 4.22, 8.1]). Let $G$ be a topological group.

(a) For each $g \in G$ there is a unique bijection $\operatorname{Ad}(g)$ of $\mathfrak{L}(G)$ onto itself such that $g X(t) g^{-1}=$ $\operatorname{Ad}(g)(X)(t)$ holds for all $t \in \mathbb{R}$.

(b) The action $G \times \mathfrak{L}(G) \rightarrow \mathfrak{L}(G):(g, X) \mapsto \operatorname{Ad}(g)(X)$ is continuous.

Now assume that $G$ has a Lie algebra.

(c) For each $g \in G$ the bijection $\operatorname{Ad}(g)$ is an automorphism of the Lie algebra $\mathbb{2}(G)$.

(d) The adjoint representation $\operatorname{Ad:~} G \rightarrow \operatorname{Aut}(\mathfrak{L}(G))$ is a continuous linear representation, where $\operatorname{Aut}(\mathfrak{L}(G))$ is endowed with the strong operator topology (i.e., the topology of pointwise convergence).

(e) The kernel of Ad is the centralizer of (the closure of) the subgroup generated by the range of the exponential function.

(f) If $G$ is a pro-Lie group then each ideal of the Lie algebra $\mathfrak{L}(G)$ is invariant under the adjoint action of the connected component of $G$.

3.4 Theorem. If $G$ is a connected pro-Lie group then $\operatorname{KOR}(G)$ is contained in the center $Z(G)$ of $G$ and is therefore abelian.

Proof. According to 3.3 the adjoint representation Ad induces ordinary representations on the finite-dimensional quotients of $\mathfrak{L}(G)$ that separate the points modulo ker Ad, which equals the center of $G$.

3.5 Corollary. For every connected locally compact group $G$ the group $\operatorname{KOR}(G)$ is contained in the center $\mathrm{Z}(G)$.

Using 2.2 and 2.6 we infer:

3.6 Corollary. The class KOR(CONNPROLIE) is closed under quotients modulo locally compact groups while $\mathrm{KOR}$ (CONNLIE) and $\mathrm{KOR}$ (CONNLCG) are closed under arbitrary (Hausdorff) quotients.

3.7 Remarks. (a) Theorem 3.4 does not extend to the case of arbitrary disconnected Lie groups even if we assume separability. In fact there are countable discrete groups $G$ with $\operatorname{KoR}(G)=G$, see 10.8 below. However, everything is fine for almost connected Lie groups, see 4.8 .

(b) There are groups in LCG $\backslash$ PROLIE such that Ad is a faithful representation (of infinite degree) but every ordinary representation is trivial on the connected component, cf. 5.2 .

The following observation will be useful to obtain restrictions on the structure and size (measured by the dimension, i.e., the rank of the Pontryagin dual) of members of KOR(CONNLCG); cf. 7.12, 7.14 and 9.1 below. The passage to the closure of the commutator group is essential, cf. 4.3 and 5.7

3.8 Lemma. (a) If $G \in$ ProLiE then $\operatorname{KOR}(G) \leq \overline{G^{\prime}}$.

(b) If $G \in \mathrm{CONNPROLIE}$ (in particular, if $G \in \mathrm{CONNLCG}$ ) then $\operatorname{KOR}(G) \leq \mathrm{Z}(G) \cap \overline{G^{\prime}}$. 
Proof. The quotient $G / \overline{G^{\prime}}$ is an abelian proto-Lie group (see [23, 4.1]). Thus $\operatorname{OR}\left(G / \overline{G^{\prime}}\right)$ separates the points, and $\operatorname{KOR}\left(G / \overline{G^{\prime}}\right)$ is trivial. For each $x \in G \backslash \overline{G^{\prime}}$ we thus find some $\rho \in \operatorname{OR}\left(G / \overline{G^{\prime}}\right)$ with $x \overline{G^{\prime}} \notin \operatorname{ker} \rho$, and composing $\rho$ with the quotient map we find $\rho^{\prime} \in \operatorname{OR}(G)$ such that $x \notin \operatorname{ker} \rho^{\prime}$.

If $G$ is also connected then $\operatorname{KoR}(G) \leq \mathrm{Z}(G)$ has been established 3.4 .

\section{Almost connected Lie groups}

4.1 Example. The following example has been around for quite some time, see [34, 4.14 , p. 191] or [35]. We use it to show that $\mathbb{R} / \mathbb{Z}$ belongs to $\operatorname{KOR}($ CONNLIE). Let $H$ be the real Heisenberg group; i.e., $H=\mathbb{R}^{3}$ with the multiplication $(a, b, x) *(c, d, y)=$ $(a+c, b+d, x+y+a d-b c)$. Clearly this is a connected Lie group, and the center $\{0\}^{2} \times \mathbb{R}$ coincides with the commutator group $H^{\prime}$.

The cyclic subgroup $Z:=\{0\}^{2} \times \mathbb{Z}$ is closed and central in $H$. Thus $H / Z$ is a connected nilpotent (and thus solvable) Lie group. According to 2.9 the compact central subgroup $C:=\left(\{0\}^{2} \times \mathbb{R}\right) / Z \cong \mathbb{R} / \mathbb{Z}$ of $(H / Z)^{\prime}$ is contained in $\operatorname{KOR}(H / Z)$. On the other hand, the quotient $H / C \cong \mathbb{R}^{2}$ has a faithful ordinary representation. Therefore, we obtain $\operatorname{KOR}(H / Z)=C \cong \mathbb{R} / \mathbb{Z}$.

For every connected Lie group $L$ with semi-simple Lie algebra one knows that $\operatorname{KOR}(L)$ is a discrete (and thus central) normal subgroup, cf. [38, 5.3.6 Thm. 8, p. 264]. In fact, for any such group one can read off $\operatorname{KOR}(L)$ from [38, Table 10, p.318f]. The relevant information is accessible algorithmically, see [4], [5]. For our present purposes, we require explicit knowledge of the case where $L$ is the simply connected covering of $\mathrm{PSL}_{2} \mathbb{R}$.

4.2 Example. Let $S$ denote the simply connected covering of $\mathrm{PSL}_{2} \mathbb{R}$. The center $\mathrm{Z}(S)$ of $S$ is infinite cyclic, but no proper covering of $\mathrm{SL}_{2} \mathbb{R}$ admits a faithful ordinary representation; see [38, Table 10, p. 318f] (where the Lie algebra $\mathfrak{s l}_{2} \mathbb{R}$ occurs in the guise of $\mathfrak{s p}_{2} \mathbb{R}=\mathfrak{s p}_{4 p+2} \mathbb{R}$ for $p=0$ ), cf. also [35] and [41, 95.9, 95.10]. Thus we obtain $\operatorname{KOR}(S)=\left\{z^{2} \mid z \in Z(S)\right\} \cong \mathbb{Z}$.

Passing from $S$ to the quotient $S_{d}:=S /\left\{z^{2 d} \mid z \in Z(S)\right\}$ we find $\operatorname{KoR}\left(S_{d}\right) \cong \mathbb{Z} / d \mathbb{Z}$ for any nonnegative integer $d$.

Instead of the simple group $\mathrm{PSL}_{2} \mathbb{R}$ we could use any other simple Lie group with infinite fundamental group (cf. 10.4) for the construction in 4.2 ,

4.3 Example. Again, let $S$ denote the simply connected covering of $\mathrm{SL}_{2} \mathbb{R}$, and let $H$ be the real Heisenberg group (cf. 4.1). Pick a generator $\zeta$ for the center of $S$. Then the subgroup $K:=\left\{\left(\zeta^{-2 z},(0,0, z)\right) \mid z \in \mathbb{Z}\right\}$ is closed and central. Passing to the quotient $G:=(S \times H) / K$ amounts to an identification of $\zeta^{2}$ with $(0,0,1)$.

Composing the inclusion maps of the two factors $S$ and $H$ with the quotient map modulo $K$ we obtain continuous homomorphisms $\eta_{S}$ and $\eta_{H}$ from $S$ and $H$, respectively, into $G$. Composition of ordinary representations of $G$ with $\eta_{X}$ then yields ordinary representations of $X \in\{S, H\}$. 
Any ordinary representation of $S$ has $\zeta^{2}$ in its kernel (cf.4.2). Therefore, any ordinary representation $\varphi$ of $G$ yields a representation $\varphi \circ \eta_{H}$ of $H$ with $(0,0,1)$ in its kernel. According to 4.1 this implies $\{0\}^{2} \times \mathbb{R} \leq \operatorname{ker}\left(\varphi \circ \eta_{H}\right)$. Therefore, the subgroup

$$
R:=\left\{\left(\zeta^{2 z},(0,0, x)\right) \mid z \in \mathbb{Z}, x \in \mathbb{R}\right\} / K
$$

is contained in $\operatorname{KOR}(G)$. Since $G / R \cong \mathrm{SL}_{2} \mathbb{R} \times \mathbb{R}^{2}$ has a faithful ordinary representation we obtain $\operatorname{KoR}(G)=R \cong \mathbb{R}$.

The examples collected so far suffice to determine the class KOR(CONNLIE). For the proof of 4.5 we need the following explicit description of the class CGAL (see also [26]).

4.4 Proposition. The elements of CGAL are precisely those of the form

$$
\mathbb{Z}^{f} \times \prod_{j \in J}\left(\mathbb{Z} / d_{j} \mathbb{Z}\right) \times(\mathbb{R} / \mathbb{Z})^{c} \times \mathbb{R}^{e}
$$

where $f, e, c$ are nonnegative integers and $\left(d_{j}\right)_{j \in J}$ is a finite family of positive integers.

Proof. The groups with a product decomposition as in $(*)$ are clearly compactly generated Lie groups. Among all locally compact abelian groups, those of the form $(*)$ are characterized by the property of being compactly generated and having no small subgroups, cf. [45, 21.17]. Since a Lie group has no small subgroups, the assertion follows.

4.5 Theorem. Exactly the compactly generated abelian Lie groups occur as $\operatorname{KOR}(L)$ for a suitable connected Lie group $L$. In other words, we have KOR(CONNLIE) = CGAL.

Proof. Each member of the class CGAL is isomorphic to a product

$$
\mathbb{Z}^{f} \times \prod_{j \in J}\left(\mathbb{Z} / d_{j} \mathbb{Z}\right) \times(\mathbb{R} / \mathbb{Z})^{c} \times \mathbb{R}^{e}
$$

where $f, e, c$ are nonnegative integers and $\left(d_{j}\right)_{j \in J}$ is a finite family of positive integers, cf. 4.4. Thus the assertion of our theorem follows from 2.7 together with the examples given in 4.1, 4.2, and 4.3. Explicitly, the group

$$
L:=S^{f} \times \prod_{j \in J} S_{d_{j}} \times(H / Z)^{c} \times((S \times H) / K)^{e}
$$

satisfies our requirements. In order to prove the converse it suffices to note that $\operatorname{KOR}(G)$ is a closed abelian subgroup of $G$ if $G \in$ CONNLIE, and thus lies in CGAL.

4.6 Remark. Even for a simply connected Lie group $L$ with simple complex Lie algebra it need not be true that $L / \operatorname{KOR}(L)$ has an irreducible faithful ordinary representation. For instance, consider the simply connected covering $\mathrm{Spin}_{8} \mathrm{C}$ of $\mathrm{SO}_{8} \mathrm{C}$; the center of that group is non-cyclic and cannot be mapped faithfully into the centralizer of an irreducible ordinary representation because that centralizer is a subgroup of the multiplicative group of Hamilton's quaternions by Schur's Lemma. However, the group $\operatorname{Spin}_{8} \mathbb{C}$ is linear, and $\operatorname{KOR}\left(\operatorname{Spin}_{8} \mathbb{C}\right)$ is trivial. 
4.7 Theorem. If $U$ is an open normal subgroup of finite index in $G$ then $\operatorname{KOR}(U)=\operatorname{KOR}(G)$.

Proof. For any $\rho \in \mathrm{OR}(U)$ the $U$-module $M_{\rho}$ associated with $\rho$ yields an induced module $L_{\rho}:=\operatorname{ind}_{U}^{G} M_{\rho}$, cf. [39, 8.4, p.230 f] or [30, XVIII 7.3]. The corresponding representation $\lambda: G \rightarrow G L\left(L_{\rho}\right)$ can now be combined with the faithful regular representation $\mu: G / U \rightarrow \mathrm{GL}\left(\mathbb{C}^{G / U}\right)$ of the finite quotient to obtain an ordinary representation of $G$ whose kernel is contained in $\operatorname{ker} \rho$. Since $\rho \in \mathrm{OR}(U)$ was arbitrary we obtain $\operatorname{KOR}(G) \leq \operatorname{KOR}(U)$. The reverse inclusion is clear from 2.2.

In an almost connected Lie group $G$ the discrete and compact quotient $G / G_{0}$ is finite. Thus 4.7yields:

4.8 Corollary. If $G$ is an almost connected Lie group then $\operatorname{KOR}(G)=\operatorname{KOR}\left(G_{0}\right)$. In particular, we have $\operatorname{KOR}($ ALMCONNLIE $)=\operatorname{KOR}($ CONNLIE $)=$ CGAL.

4.9 Remark. One would of course like to extend 4.8 to the classes ALMCONNLCG $\subset$ ALMCONNPROLIE. The ordinary representations of the compact quotient separate the points; thus $\operatorname{KOR}(G) \leq G_{0}$ if $G \in$ ALMCONNPROLIE. If one wants to proceed along the lines of the proof of 4.7 then there remains the problem to extend a representation of $G_{0}$ via induction. This question is treated by Mackey [32] where an invariant scalar product is assumed and the group in question is required to be locally compact and separable. Note that 4.7 yields $\operatorname{KOR}(U)=\operatorname{KOR}(G)$ for each open normal subgroup of $G$ but $\operatorname{KOR}\left(G_{0}\right)$ might still be smaller although $G_{0}$ is the intersection of those open normal subgroups (cf. [45, 6.8]).

\section{Examples that are not Lie groups}

A natural source of locally compact groups that are not Lie groups originates from the fact that the class CG is closed under arbitrary cartesian products. Another wellunderstood (and rich) class of locally compact groups is the class LCA. Both classes do not contribute to KOR(LCG):

5.1 Theorem. The class $\mathrm{KOR}(\mathrm{CG}) \cup \mathrm{KOR}(\mathrm{LCA}) \cup \mathrm{KOR}(\mathrm{ABPROLIE})$ consists of the trivial group alone. In fact $\operatorname{KOR}(G)$ is trivial for every abelian proto-Lie group $G$.

Proof. The Peter-Weyl Theorem asserts that the ordinary representations separate the points in any compact group, cf. [45, 14.33]. Thus KOR(CG) contains only the trivial group.

It remains to consider $A \in \mathrm{LCA}$. Every character of $A$ is a continuous homomorphism from $A$ into $\mathbb{R} / \mathbb{Z} \cong \mathrm{SU}_{1} \mathbb{C}<\mathrm{GL}_{1} \mathbb{C}$ and thus belongs to $\mathrm{OR}(A)$. Pontryagin duality (cf. [45, 22.6]) implies that the characters separate the points of $A$. Thus $\operatorname{KOR}(A)$ is trivial.

For a proto-Lie group $G$ the continuous homomorphisms to Lie groups separate the points. If $G$ is abelian then it suffices to consider homomorphisms from $G$ to abelian Lie groups, and it follows that $\operatorname{KOR}(G)$ is trivial. 
5.2 Example. Let $C$ be a compact simple non-abelian group and let $D$ be any infinite discrete group. For instance, the group $C=\mathrm{SO}_{3} \mathbb{R}$ would do - in any case, $C$ will be connected (see [45, 4.13]). The product topology turns $C^{D}$ into a compact connected group belonging to CONNLCG $\subset$ CONNPROLIE but not to LIE. We form the semidirect product $G:=D \ltimes C^{D}$ where conjugation with $d \in D$ maps $\left(c_{j}\right)_{j \in D} \in C^{D}$ to $\left(b_{j}\right)_{j \in D}$ with $b_{j}=c_{d j}$. Then $G \in$ LCG.

The closed normal subgroups of $C^{D}$ are in one-to-one correspondence with the lattice of subsets of $D$, cf. [44, 3.12]: to $J \subseteq D$ we associate the product $\prod_{j \in D} B_{j}$ where $B_{j}=C_{j}$ if $j \in J$ and $B_{j}$ is trivial if $j \in D \backslash J$. Consequently, every non-trivial closed normal subgroup of $G$ contains the connected component $G_{0} \cong C^{D}$, and we have $\operatorname{Kor}(G)=$ $G_{0} \cong C^{D}$ here.

Our investigation of the normal subgroups also makes clear that $G$ does not belong to PROLIE.

5.3 Example. Let $S$ again denote the universal covering group of $\mathrm{SL}_{2} \mathbb{R}$. As in [24, Ex. 0.6] we consider the filter basis $\mathcal{N}(S)^{\times}$of all nontrivial subgroups of the center of $S$. The projective limit $G:=\lim _{N \in \mathcal{N}(S) \times S / N}$ is a connected locally compact group (see [24, 2.12]) with a center $\mathrm{Z}(G)$ isomorphic to the universal zero dimensional compactification of $\mathbb{Z}$.

In other words $Z(G)$ is isomorphic to $\prod_{p \in \mathbb{P}} \mathbb{Z}_{p}$ where $\mathbb{P}$ is the set of primes and $\mathbb{Z}_{p}$ is the additive group of $p$-adic integers. Note that $Z(G)$ has a unique quotient of order 2 because $Z(G) /\left\{z^{2} \mid z \in Z(G)\right\} \cong \prod_{p \in \mathbb{P}} \mathbb{Z}_{p} / \prod_{p \in \mathbb{P}} 2 \mathbb{Z}_{p} \cong \mathbb{Z}_{2} / 2 \mathbb{Z}_{2} \cong \mathbb{Z} / 2 \mathbb{Z}$. From [24, 2.14] we infer that $G$ and $G / Z(G) \cong P L_{2} \mathbb{R}$ have essentially the same Lie algebra.

As $G / Z(G) \cong \mathrm{PSL}_{2} \mathbb{R}$ is a simple group, the only proper normal subgroups of $G$ are those of $Z(G)$. If $\rho$ is an ordinary representation of $G$ then $\rho(Z(G))$ is a pro-finite subgroup of a Lie group and thus finite. This means that $\operatorname{ker} \rho=\operatorname{ker}\left(\left.\rho\right|_{Z(G)}\right)$ is co-finite in $Z(G)$, and $G / \operatorname{ker} \rho$ is an extension of Lie groups (namely $G / Z(G) \cong P L_{2} \mathbb{R}$ and the finite group $Z(G) / \operatorname{ker} \rho$ ). Now $G / \operatorname{ker} \rho$ is a connected Lie group, has the same Lie algebra as $\mathrm{PSL}_{2} \mathbb{R}$ and possesses a faithful ordinary representation. Thus $|Z(G) / \operatorname{ker} \rho| \leq$ 2 and $\operatorname{ker} \rho$ contains $\left\{z^{2} \mid z \in Z(G)\right\} \cong \prod_{p \in \mathbb{P}} \mathbb{Z}_{p}$. Since $G /\left\{z^{2} \mid z \in Z(G)\right\} \cong \mathrm{SL}_{2} \mathbb{R}$ clearly has a faithful ordinary representation we obtain $\operatorname{KOR}(G) \cong \prod_{p \in \mathbb{P}} \mathbb{Z}_{p}$.

5.4 Definitions. A topological group $G$ is called monothetic if there exists $g \in G$ such that the closure of the subgroup generated by $g$ is dense in $G$. Any such $g$ is called a topological generator of $G$. The class of all compact monothetic (necessarily abelian) groups will be denoted by MONCA.

The one-parameter subgroups of $G$ are the elements of $\operatorname{Hom}(\mathbb{R}, G)$. We say that $G$ has a dense one-parameter subgroup if there is $\varphi \in \operatorname{Hom}(\mathbb{R}, G)$ with $\overline{\varphi(\mathbb{R})}=G$.

5.5 Lemma. (a) A locally compact monothetic group is either compact or isomorphic to $\mathbb{Z}$.

(b) If $A \in \mathrm{LCA}$ has a dense one-parameter subgroup then $A$ is either isomorphic to $\mathbb{R}$ or $A$ is a connected compact monothetic group.

(c) The Pontryagin duals of compact monothetic groups are the subgroups of the discrete group $\mathbb{Q}^{\left(2^{\aleph_{0}}\right)} \times \mathbb{Q} / \mathbb{Z}$. 
(d) The duals of connected compact monothetic groups are the subgroups of the discrete group $\mathbb{Q}^{\left(2^{\aleph_{0}}\right)} \cong \mathbb{R}_{\text {discr. }}$.

Proof. The first two assertions are known as Weil's Lemma, cf. [45, 6.26]. Assertion[(c) is due to [11], we present the argument for the reader's convenience. The existence of a cyclic subgroup means that there is an epimorphism $\eta: \mathbb{Z} \rightarrow A$ in the category LCA which upon dualizing gives a monomorphism $\eta^{*}: A^{*} \rightarrow \mathbb{Z}^{*} \cong \mathbb{R} / \mathbb{Z}$; cf. [45, 15.5, 15.7, 20.13]. Since $A$ is compact the dual $A^{*}$ is discrete (see. [45, 20.6]), and we may interpret $\eta^{*}$ as an embedding of $A^{*}$ into the discrete group $\mathbb{R}_{\text {discr }} / \mathbb{Z} \cong \mathbb{Q}^{\left(2^{\aleph_{0}}\right)} \times \mathbb{Q} / \mathbb{Z}$. Now connectedness of $A$ implies that $A^{*}$ is torsion-free (cf. [45, 23.18]) and $\eta^{*}$ induces an embedding of $A^{*}$ into the quotient $\mathbb{Q}^{\left(2^{\aleph_{0}}\right)}$ of $\mathbb{R}_{\text {discr }} / \mathbb{Z}$ modulo its torsion group $\mathbb{Q} / \mathbb{Z}$.

In order to prove the last assertion we dualize the epimorphism $\varphi: \mathbb{R} \rightarrow A$ to a monomorphism $\varphi^{*}: A^{*} \rightarrow \mathbb{R}^{*} \cong \mathbb{R}$ and then replace the range by the discrete group $\mathbb{R}_{\text {discr. }}$.

5.6 Example. Generalizing the construction described in 4.1 we take $A \in$ LCA with a dense one-parameter group $\varphi \in \operatorname{Hom}(\mathbb{R}, A)$ and define a multiplication $*$ on $\mathbb{R}^{2} \times A$ by $(a, b, x) *(c, d, y)=(a+c, b+d, x+y+\varphi(a d-b c))$. Then $H_{\varphi}:=\left(\mathbb{R}^{2} \times A, *\right)$ is a connected locally compact group. If $A$ is compact we proceed as in 4.1 to see $\operatorname{KOR}\left(H_{\varphi}\right)=\{0\}^{2} \times A \cong A$.

5.7 Example. Again, let $S$ denote the universal covering group of $\mathrm{SL}_{2} \mathbb{R}$. Assume that $A$ is a compact monothetic group and let $c$ be a topological generator. Proceeding as in 4.3 we construct the quotient $G$ of $S \times A$ such that $\zeta^{2}$ is identified with $c$. Then an argument as in 4.3 shows that $\operatorname{KOR}(G)$ contains the closure of $c$ in $G$. This is a subgroup isomorphic to $A$.

\section{Connected pro-Lie groups}

In order to show $\mathrm{CA} \subset \mathrm{KOR}(\mathrm{CONNPROLIE})$ we study free compact abelian groups.

6.1 Definitions. For a pointed compact space $X$ let $X /$ conn be the totally disconnected compact space of connected components of $X$ and let $\mathfrak{w}(X)$ denote the weight of $X$ (i.e., the minimal cardinality of a basis for the topology on $X)$. Put $\mathfrak{w}_{0}(X):=\mathfrak{w}(X)-1$; this coincides with $\mathfrak{w}(X)$ if the latter is infinite.

Let $C_{0}(X, \mathbb{T})$ denote the set of all continuous functions from $X$ to $\mathbb{T}$ mapping the base point to 0 . This set endowed with the compact-open topology and the pointwise operations becomes a topological group. The quotient $[X, \mathbb{T}]:=C_{0}(X, \mathbb{T}) / C_{0}(X, \mathbb{T})_{0}$ modulo the connected component $C_{0}(X, \mathbb{T})_{0}$ is discrete (cf. the paragraph preceding [22, 8.50]); its compact Pontryagin dual $[X, \mathbb{T}]^{*}$ plays a crucial role in the structure of the free compact abelian group $F(X)$.

6.2 Theorem. For every nonsingleton pointed compact space the free compact abelian group $F(X)$ is isomorphic to $\left(\mathbb{Q}^{*}\right)^{\mathfrak{w}(X)^{\aleph_{0}}} \times \prod_{p \in \mathbb{P}} \mathbb{Z}_{p}^{\mathfrak{w}_{0}(X / \text { conn })} \times[X, \mathbb{T}]^{*}$. The group $[X, \mathbb{T}]$ is torsionfree, and its Pontryagin dual $[X, \mathbb{T}]^{*}$ is a quotient of some power of $\mathbb{Q}^{*}$. 
Proof. The structure of $F(X)$ is known from [18, 1.5.4], cf. [22, 8.67]. For every compact Hausdorff space $X$ the group $[X, \mathbb{T}] \cong H^{1}(X, \mathbb{Z})$ is torsion-free, see [18, 1.3.1], cf. [22. 8.50(ii)]. For $d:=\operatorname{dim}_{\mathbb{Q}}(\mathbb{Q} \otimes[X, \mathbb{T}])$ we have an embedding of $[X, \mathbb{T}]$ into $\mathbb{Q} \otimes[X, \mathbb{T}] \cong$ $\mathbb{Q}^{(d)}$ which dualizes to the quotient map in question.

6.3 Theorem. The class $\mathrm{KOR}(\mathrm{CONNPROLIE})$ contains at least the class $\Pi(\mathrm{CGAL} \cup \mathrm{CA})=$ $\Pi(\{\mathbb{Z}, \mathbb{R}\} \cup \mathrm{CA})$. In particular, we have CONNABProLie $\subset$ Kor(CONNPROLie).

The class $\operatorname{KOR}($ CONNLCG) contains the class SMALLLCA $=\mathbf{P}(\{\mathbb{Z}, \mathbb{R}\} \cup$ MONCA $)$ consisting of all groups of the form $\mathbb{Z}^{f} \times A \times \mathbb{R}^{e}$ with $e, f \in \mathbb{N}$ and a compact monothetic group $A$.

Proof. Abelian pro-Lie groups are studied in [21], cf. [23, Ch. 5]: in particular, the connected ones are of the form $\mathbb{R}^{c} \times C$ where $c$ is arbitrary (possibly infinite) and $C$ is a connected compact abelian group. Such a group belongs to CONNLCA precisely if $c$ is finite, cf. [45, 24.9].

Let $C$ be a compact abelian group. Then $C$ is a quotient of the free compact abelian group $F(C)$. From 6.2 we know that $F(C)$ is a quotient of a product of compact monothetic groups. The class MONCA of all compact monothetic groups is contained in KOR $($ CONNLCG $) \subset$ KOR (CONNPROLIE) by 5.7and 5.6. From 2.7and 3.6 we conclude $\mathrm{CA}=\mathbf{Q \Pi}(\mathrm{MONCA}) \subseteq \mathrm{KOR}(\mathrm{CONNPROLIE})$ and $\mathbf{Q P}(\mathrm{MONCA}) \subseteq \mathrm{KOR}(\mathrm{CONNLCG})$

From 4.3 we recall that $\mathbb{Z}$ and $\mathbb{R}$ lie in $\operatorname{Kor}($ CONNLCG $) \subset \mathrm{KOR}($ CONNPROLIE); it remains to use products once again.

6.4 Remark. The factor $A$ in 6.3 cannot be arbitrarily large; we show $\mathfrak{w}\left(A_{0}\right) \leq 2^{\aleph_{0}}$ in 7.14 below. If the answer to 9.1 is affirmative then we know that SMALLLCA coincides with $\operatorname{Kor}($ CONNLCG), cf.7.15.

6.5 Remarks. We have mentioned in 6.2 that the factor $[X, \mathbb{T}]^{*}$ of $F(X)$ has a torsion-free dual. Conversely, every torsion-free abelian group $A$ is isomorphic to $[X, \mathbb{T}]$ for some compact connected Hausdorff space (namely, for the underlying space $X=\left|A^{*}\right|$ of the Pontryagin dual of $A$ ), see [18, 1.3.2]. The corrections in [19] only concern assertions about cardinalities (dimension, rank) in [18].

6.6 Proposition. Each discrete member of KOR(CONNPROLIE) is finitely generated. Each member of $\mathrm{KOR}$ (CONNLCG) is compactly generated.

Proof. Discrete central subgroups of connected pro-Lie groups are finitely generated by [25, 5.10]. Consider $G \in$ CONNLCG. Any compact neighborhood in $\operatorname{KOR}(G)$ generates a central subgroup $C$ of $G$, and $\operatorname{KoR}(G) / C$ is a discrete subgroup of $G / C \in$ CONNLCG $\subset$ CONNPROLiE. Now it remains to note that the class of compactly generated locally compact groups is closed under extensions, see [45, 6.11].

6.7 Remark. Locally compact abelian groups are compactly generated precisely if they are contained in some almost connected locally compact group, see [26, Cor.1]. The compactly generated members of LCA are of the form $\mathbb{Z}^{f} \times C \times \mathbb{R}^{e}$ with natural numbers $e, f$ and some $C \in \mathrm{CA}$, cf. [45, 23.11]. Note that every $C \in \mathrm{CA}$ is contained in 
a connected compact group because the characters separate the points: this yields an embedding into $\mathbb{T}^{C^{*}}$.

\section{Connected locally compact groups}

Our aim in this section is to establish a bound on the size of $\operatorname{KOR}(G)$ if $G \in$ CONNLCG. To this end, we need some information on the weight (cf.6.1) and generating rank.

If $X$ is discrete then $\mathfrak{w}(X)$ is just the cardinality $|X|$. The function $\mathfrak{w}$ is monotonic; i.e. each subspace $Y \subseteq X$ satisfies $\mathfrak{w}(Y) \leq \mathfrak{w}(X)$. For an infinite compact group the weight coincides with the local weight, i.e., the minimal cardinality of a neighborhood basis.

For $C \in$ CONNCA a finer invariant than the weight is the rank $\operatorname{dim}_{\mathbb{Q}}\left(\mathbb{Q} \otimes C^{*}\right)$ of its dual $C^{*}$. Note that $C^{*}$ embeds in $\mathbb{Q} \otimes C^{*}$ only if $C^{*}$ is torsion-free (i.e., if $C$ is connected, see [45, 23.18]). The rank of $C^{*}$ coincides with the topological dimension of $C$, cf. [22, 8.26]. Every compact abelian Lie group has finite dimension; its dual is finitely generated.

7.1 Lemma. (a) For each positive integer $n$ we have $\mathfrak{w}\left(\mathbb{R}^{n}\right)=\aleph_{0}$.

(b) The equality $\mathfrak{w}(A)=\mathfrak{w}\left(A^{*}\right)$ holds for each $A \in$ LCA.

(c) In particular, for $C \in \mathrm{CA}$ we have $\mathfrak{w}(C)=\left|C^{*}\right|$.

(d) For $C \in C A$ we have $\mathfrak{w}\left(C_{0}\right)=\max \left\{\aleph_{0}, \operatorname{dim}_{\mathbb{Q}}\left(\mathbb{Q} \otimes C^{*}\right)\right\}$ unless $C$ is trivial.

(e) If $n$ is a positive integer and $C \in$ CONNCA then $\mathfrak{w}\left(\mathbb{R}^{n} \times C\right)=\max \left\{\aleph_{0}, \mathfrak{w}(C)\right\}$.

(f) If $G \in C G$ and $N$ is a totally disconnected closed normal (and thus central) subgroup of $G_{0}$ then $\mathfrak{w}(G)=\mathfrak{w}(G / N)$.

Proof. The assertion on $\mathfrak{w}\left(\mathbb{R}^{n}\right)$ is obvious from the fact that the underlying space is metrizable and the weight equals the local weight. See [15, 24.14] or [22, 7.76] for assertion (b)] The assertions on compact groups are taken from [22, 12.25] and [20, 3.2].

7.2 Lemma. For $C \in \mathrm{CA}$ we have $\mathfrak{w}\left(C_{0}\right) \leq 2^{\aleph_{0}}$ precisely if $C_{0}$ is monothetic. If $C \in \mathrm{CA}$ has a finitely generated dense subgroup then $\mathfrak{w}(\bar{C}) \leq 2^{\aleph_{0}}$.

Proof. From 7.1 we know $\mathfrak{w}\left(C_{0}\right)=\operatorname{dim}_{\mathbb{Q}}\left(\mathbb{Q} \otimes C_{0}^{*}\right)$. Since $C_{0}^{*}$ is torsion-free we have an embedding $\eta: C_{0}^{*} \rightarrow \mathbb{Q} \otimes C_{0}^{*}$. Thus $C_{0}^{*}$ is the dual of a monothetic group, see 5.5.

7.3 Suitable sets and generating rank. Following [20] (cf. [22, 12.1]), a subset $X$ of a topological group $G$ is called suitable if it does not contain the neutral element 1, is discrete and closed in $G \backslash\{1\}$, and generates a dense subgroup of $G$. Every locally compact group possesses suitable sets by [20,1.12]. We define the generating rank $s(G)$ as the minimum over the cardinalities of suitable sets.

7.4 Examples. (a) For the additive group $\mathbb{R}$ any two elements that are linearly independent over $\mathbb{Q}$ form a suitable set. Thus $s(\mathbb{R})=2$.

(b) A suitable set for $\mathbb{R}^{n}$ needs at least $n+1$ elements because fewer vectors will either be linearly dependent (and thus contained in a proper closed subgroup) or form a basis (and then generate a discrete proper subgroup). It is known that there exists 
$v \in \mathbb{R}^{n}$ such that $v+\mathbb{Z}$ generates a dense subgroup of $\mathbb{R}^{n} / \mathbb{Z}^{n}$. The standard basis together with any such $v$ forms a suitable set for $\mathbb{R}^{n}$. Thus $s\left(\mathbb{R}^{n}\right)=n+1$.

(c) For $G \in$ CONNCG the generating rank depends on the weight, see [22, 12.22]:

(i) If $\mathfrak{w}(G) \leq 2^{\aleph_{0}}$ and $G$ is abelian (but not trivial) then $s(G)=1$.

(ii) If $\mathfrak{w}(G) \leq 2^{\aleph_{0}}$ and $G$ is not abelian then $s(G)=2$.

(iii) If $\mathfrak{w}(G)>2^{\aleph_{0}}$ then $s(G)=\min \left\{\beth \mid \mathfrak{w}(G) \leq \beth^{\aleph_{0}}\right\}$.

(d) In particular, the generating rank of any connected Lie group is finite.

7.5 Lemma ([22, 12.26]). Let $G \in$ CONNCG and let $N$ be a normal subgroup of $G$. Then $s(G / N) \leq s(G) \leq s(G / N)+s(N)$. If $N$ is totally disconnected then $s(G)=s(G / N)$.

7.6 Reduction to maximal compact subgroups. Let $G \in$ ALMCONNLCG. By the Mal'tsev-Iwasawa Theorem (see [28, Thm.13] combined with the solution of Hilbert's Fifth Problem [49], cf. [34] or [29]) there exists a maximal compact subgroup $M$ of $G$ which is unique up to conjugacy. If $M \neq G$ then there is a positive integer $n$ (called the characteristic index in [28]) and there are subgroups $R_{1}, \ldots, R_{n}$ all isomorphic to $\mathbb{R}$ such that $G=R_{1} \cdots R_{n} M$. The number $n$ equals the topological dimension $\operatorname{dim} G / M$ of the coset space $G / M$ (which is actually a manifold). If $M=G$ we just have $n=0$.

Note that $M$ is connected. Thus the weight of $G$ equals that of $M$ if $G=M$ and satisfies $\mathfrak{w}(G)=\max \left\{\aleph_{0}, \mathfrak{w}(M)\right\}$ otherwise.

7.7 Proposition. For $G \in$ ALMCONNLCG pick a maximal compact subgroup $M$ of $G$. Then $s(G) \leq 2 \operatorname{dim} G / M+s(M)$. In particular, the generating rank $s(G)$ is finite if $\mathfrak{w}(M) \leq 2^{\aleph_{0}}$. If $\psi: G \rightarrow H$ is a continuous homomorphism with dense range then $s(G) \geq s(H)$.

Proof. We use subgroups $R_{1}, \ldots, R_{n}$ as in 7.6, where $n:=\operatorname{dim} G / M$. Combining a suitable set for $M$ with suitable sets for each $R_{j}$ we find $s(G) \leq 2 n+s(M)$. From 7.4 we thus infer that $s(G)$ is finite if $G \in$ CONNLCG satisfies $\mathfrak{w}(G) \leq 2^{\aleph_{0}}$.

Every suitable set $X$ constructed in this way will be relatively compact because only finitely many elements lie outside the compact group $M$. Now [22, 12.4] asserts that $\psi(X) \backslash\{1\}$ will be suitable in $H$ whenever $\psi: G \rightarrow H$ is a continuous homomorphism with dense range.

7.8 Lemma. For each non-trivial $G \in$ ALMCONNLCG we have $s(G) \leq \mathfrak{w}(G) \leq s(G)^{\aleph_{0}}$.

Proof. By the Mal'tsev-Iwasawa Theorem (cf. (7.6) we know that $G$ is homeomorphic to $\mathbb{R}^{n} \times M$ for a maximal compact subgroup $M$ and some nonnegative integer $n$. The estimates $s(M) \leq \mathfrak{w}(M) \leq s(M)^{\aleph_{0}}$ are valid for any non-trivial compact group $M$, see [22, 12.27].

If the group $G$ is compact it coincides with $M$. If $M$ is trivial but $n \geq 1$ then 7.7yields that $s(G) \leq 2 n$ is finite. Thus $s(G) \leq \aleph_{0}=\mathfrak{w}(G)<2^{\aleph_{0}}=s(G)^{\aleph_{0}}$.

There remains the case where $G$ is not compact and $M$ is not trivial. Then $n \geq 1$ and $\mathfrak{w}(G)=\max \left\{\aleph_{0}, \mathfrak{w}(M)\right\}$. Now the estimates $s(G) \leq 2 n+s(M) \leq \aleph_{0}+s(M) \leq$ $\aleph_{0}+\mathfrak{w}(M)=\mathfrak{w}(G)$ from 7.7 and $\mathfrak{w}(G)=\aleph_{0}+\mathfrak{w}(M) \leq \aleph_{0}+s(M)^{\aleph_{0}} \leq 2^{\aleph_{0}}+s(M)^{\aleph_{0}}=$ $s(G)^{\aleph_{0}}$ yield the claim. 
7.9 Remark. The inequality $s(G) \leq \mathfrak{w}(G)$ holds for arbitrary $G \in$ LCG see [20, 4.2].

7.10 Lemma. Let $G \in$ CONNLCG. We consider a compact normal subgroup $K$ of $G$, the centralizer $C:=C_{G}(K)$ and its connected component $C_{0}$. Then $G=C_{0} K$.

Proof. We abbreviate $D:=C \cap K$. From [28, Thm. 2] we know $C K=G$. The group $C$ is a closed subgroup of the $\sigma$-compact group $G$ and thus $\sigma$-compact itself, see [45, 6.10, 6.12]. The Open Mapping Theorem (cf. [45, 6.19]) yields that $C / D$ is isomorphic to $G / K$. Now $C /\left(C_{0} D\right)$ is totally disconnected (cf. [45, 6.9]) but also connected because it is a continuous image of $C / D \cong G / K$. Thus $C=C_{0} D$ and $C_{0} K=C_{0} D K=C K=G$.

7.11 Theorem. If $G \in$ CONNLCG has a compact normal subgroup $K$ such that $G / K$ is a Lie group and $K_{0}$ is abelian then $s\left(\overline{G^{\prime}}\right)$ is finite. Consequently, the group $\operatorname{KOR}(G)$ satisfies $\mathfrak{w}(\operatorname{KOR}(G)) \leq 2^{\aleph_{0}}$ in this case.

Proof. The compact abelian normal subgroup $K_{0}$ is contained in the center of $G$. A dense subgroup of $\overline{G^{\prime}}$ is generated by $\gamma(S \times S)$ where $S$ is any suitable set for $G / K_{0}$ and $\gamma: G / K_{0} \times G / K_{0} \rightarrow \overline{G^{\prime}}:\left(a K_{0}, b K_{0}\right) \mapsto a b a^{-1} b^{-1}$ is induced by the commutator map.

We pick a maximal compact subgroup $M$ of $G$. Then $K_{0} \leq M$ and by 7.7 there exists a nonnegative integer $n$ such that $s\left(G / K_{0}\right) \leq 2 n+s\left(M / K_{0}\right)$. Now $s\left(G / K_{0}\right)$ is finite because the compact connected Lie group $M / K$ has finite generating rank and $s\left(M / K_{0}\right)=s(M / K)$ by 7.5 .

Thus we may choose a finite set $S$; then $\gamma(S \times S) \backslash\{1\}$ is a finite (and thus indeed closed and discrete) suitable set for $\overline{G^{\prime}}$. The bound for the weight of $\operatorname{KOR}(G)$ now follows from 7.8 , monotonicity of the weight function and the fact that $\operatorname{KOR}(G)$ is contained in $\overline{G^{\prime}}$, see 3.8 .

For any solvable compact group the connected component is abelian, see [28, Thm. 2]. Thus 7.11 yields:

7.12 Corollary. If $G$ is a solvable connected locally compact group then $s\left(\overline{G^{\prime}}\right)<\aleph_{0}$ and $\mathfrak{w}(\operatorname{KOR}(G)) \leq \mathfrak{w} \overline{G^{\prime}} \leq 2^{\aleph_{0}}$.

7.13 Example. Let $\aleph$ be any cardinal, let $S$ be a connected compact Lie group with simple Lie algebra and non-trivial center $Z$, and put $G:=S^{\aleph}$. Then $Z$ is finite and $\overline{S^{\prime}}=S$. Thus $G$ is a compact connected group with $\overline{G^{\prime}}=G$ and $Z(G) \cap \overline{G^{\prime}}=Z(G) \cong Z^{\aleph}$. Now $\mathfrak{w}\left(Z(G) \cap \overline{G^{\prime}}\right)=\mathfrak{w}\left(Z^{\aleph}\right)=\left|Z^{(\aleph)}\right|$, and this cardinality may be arbitrarily large.

7.14 Proposition. For each $G \in$ CONNLCG we have $\mathfrak{w}\left(\operatorname{KOR}(G)_{0}\right) \leq 2^{\aleph_{0}}$. In particular, the connected component of the maximal compact subgroup of $\operatorname{KOR}(G)$ is monothetic.

Proof. Let $K$ be the maximal compact normal subgroup of $G$ (cf. [28, Thm. 14]). Then 7.10 says $G=C_{0} K$ where $C_{0}$ is the connected component of the centralizer $C:=C_{G}(K)$ of $K$ in $G$. Thus $\overline{G^{\prime}}=\overline{C_{0}^{\prime} K^{\prime}}=\overline{C_{0}^{\prime}} \overline{K^{\prime}}$ because $\overline{K^{\prime}}$ is compact, and $\left(Z(G) \cap \overline{G^{\prime}}\right)_{0} \leq C_{0} \cap \overline{G^{\prime}}$ is contained in $\overline{C_{0}^{\prime}}\left(C_{0} \cap \overline{K^{\prime}}\right) \leq C_{0}$. 
The locally compact group $C_{0} /\left(C_{0} \cap K\right)$ admits an injective continuous homomorphism into the Lie group $G / K$ and thus is a Lie group itself. Moreover, we have that $C_{0} \cap K$ is contained in the center of $K$ and thus abelian. Thus 7.11 applies to $C_{0}$ and $K$, yielding $\mathfrak{w}\left(\overline{C_{0}^{\prime}}\right) \leq 2^{\aleph_{0}}$.

The group $B:=C_{0} \cap \overline{K^{\prime}}$ is contained in the intersection of $\overline{K^{\prime}}$ with the center of the compact group $K$. Thus $B$ is totally disconnected, see [22, 9.23], and the quotient $B \overline{C_{0}^{\prime}} / \overline{C_{0}^{\prime}} \cong B /\left(B \cap \overline{C_{0}^{\prime}}\right)$ is totally disconnected, as well. This yields that the connected component $\operatorname{KoR}(G)_{0}$ of $\operatorname{KoR}(G)$ is contained in $\overline{C_{0}^{\prime}}$, and the bound $\mathfrak{w}\left(\operatorname{KOR}(G)_{0}\right) \leq 2^{\aleph_{0}}$ is established. Now the connected component of the maximal compact subgroup of $\operatorname{KOR}(G)$ is monothetic by 7.2 .

7.15 Theorem. For each $G \in \mathrm{CONNLCG}$ there exist $A \in \mathrm{CA}$ and natural numbers $e, f$ such that $A_{0}$ is monothetic and $\operatorname{KOR}(G) \cong \mathbb{Z}^{f} \times A \times \mathbb{R}^{e}$. In particular, the dimension of members of $\mathrm{KOR}(\mathrm{CONNLCG})$ is bounded by $2^{\aleph_{0}}$.

Proof. We combine 7.14 with 6.6, 6.7, and 7.2 ,

\section{Partial results}

The classes Kor(Seplie), Kor(Lie), Kor(LCG) and Kor(Prolie) are quite large and not very well understood. We will indicate some large subclasses and note (in 8.4) that these classes are not closed under the operations $\overline{\mathbf{S}}, \mathbf{Q}$, and $\widehat{\mathbf{Q}}$.

8.1 Theorem. The class KOR(SEPLIE) contains the following:

(a) The class KOR(CONNLIE) = KOR(ALMCONNLIE) = CGAL, cf. 4.5 and 4.8

(b) All countable discrete simple groups with infinite elementary abelian subgroups, $c f$.10.8(a)

(c) Countably infinite discrete simple groups with finitely many conjugacy classes, such as those constructed as HNN-extensions, see 10.8(b).

Moreover, KOR(SEPLIE) is closed under $\mathbf{P}$.

Note that KOR(SEPLIE) is considerably larger than KOR(ALMCONNLIE) = CGAL.

8.2 Theorem. The class KOR(LCG) contains the following:

(a) The class KOR(SEPLIE) $\cup$ KOR(CONNLCG), and thus CGAL and all compact monothetic groups.

(b) All groups of the form $C^{D}$ where $C$ is a compact simple non-abelian group and $D$ is infinite, see 5.2

(c) All simple non-discrete totally disconnected locally compact groups.

(d) All simple discrete groups of cardinality larger than $2^{\aleph_{0}}$.

(e) All discrete simple groups with infinite elementary abelian subgroups.

Moreover, KOR(LCG) is closed under $\mathbf{P}$ but not under $\boldsymbol{\Pi}$.

The class in 8.2|(c) includes the simple $p$-adic groups such as $\operatorname{PSL}_{n} \mathbb{Q}_{p}$. Among the groups in 8.2(d) we find, for instance, the simple classical groups over large fields such as $\operatorname{PSL}_{n} F$ where $F=Q(X)$ is a purely transcendental extension with a transcendency basis $X$ such that $|X|>|\mathbb{R}|$. 
8.3 Proposition. The groups $\mathrm{SO}_{3} \mathbb{R}$ and $\mathrm{PSL}_{2} \mathbb{C}$ do not belong to $\mathrm{KOR}(\mathrm{TG})$.

Proof. The Lie algebra $\mathfrak{L}\left(\mathrm{SO}_{3} \mathbb{R}\right)$ is isomorphic to the vector product algebra $\left(\mathbb{R}^{3}, \times\right)$, and $\mathrm{Ad}\left(\mathrm{SO}_{3} \mathbb{R}\right) \cong \mathrm{SO}_{3} \mathbb{R}$ contains all automorphisms of that algebra. Therefore, each automorphism of $\mathrm{SO}_{3} \mathbb{R}$ is an inner automorphism, see2 [22, 6.59].

Now assume that there exists a group $G \in \mathrm{TG}$ with $\operatorname{KOR}(G) \cong \mathrm{SO}_{3} \mathbb{R}$. Then $G$ is the direct product of $\operatorname{KoR}(G)$ with its centralizer $C$, see 7.10. Thus $G / C \cong \mathrm{SO}_{3} \mathbb{R}$ has a faithful ordinary representation, and $\operatorname{KOR}(G) \leq C$. This is a contradiction.

For the group $\mathrm{PSL}_{2} \mathrm{C} \cong \mathrm{PGL}_{2} \mathrm{C}$ we can proceed in the same way because this group also has only inner automorphisms, see [42], cf. [10].

8.4 Corollary. The classes KOR(LCG) and KOR(ProLiE) are not closed under any one of the operations $\overline{\mathbf{S}}, \mathbf{Q}$, or $\widehat{\mathbf{Q}}$.

8.5 Remark. The group $\mathrm{PSL}_{2} \mathbb{R}$ has outer automorphisms (induced by elements of $\mathrm{GL}_{2} \mathbb{R}$ with non-square determinant). Every group $\mathrm{PSL}_{n} F$ with $n>2$ over a commutative field $F$ has outer automorphisms induced by polarities of the projective space. Thus the argument used in the proof of 8.3 does not easily extend to arbitrary classical simple groups.

\section{Open questions}

9.1 Problem. Is it true that $\mathfrak{w}(\operatorname{KOR}(G)) \leq 2^{\aleph_{0}}$ holds for every $G \in$ CONNLCG ?

Comments on 9.1. If the answer to this problem is affirmative then KOR(CONNLCG) = SMALLLCA, cf. 6.3, 6.6 and 6.7.

In 6.6 we have seen that KOR(CONNLCG) consists of compactly generated groups, and 7.14 says that the connected component of the maximal compact subgroup of $\operatorname{KOR}(G)$ lies in SMALLCA. For an affirmative answer to 9.1 it suffices to exclude totally disconnected compact groups $A$ with $\mathfrak{w}(A)>2^{\aleph_{0}}$ from KOR(CONNLCG) because that class is closed under the passage to Hausdorff quotients, cf. 2.5.

For $G \in$ CONNLCG consider the maximal compact normal subgroup $K$ of $G$ and let $C_{0}$ be the connected component of the centralizer of $K$. Then the weight of $C_{0} \cap \overline{K^{\prime}}$ may be arbitrarily large, as 7.13 shows. It is therefore clear that we have to find a more subtle approach than 3.8 if we want to give an affirmative answer to 9.1 .

9.2 Problem. Which abelian pro-Lie groups are in KOR(CONNPROLIE) ?

Comments on 9.2. Every element of KOR(CONNPROLIE) is contained in the center of a connected pro-Lie group (namely, $G$ ) and thus contained in some connected abelian pro-Lie group, cf. [23, 12.90]. From 6.3] we thus infer

$$
\text { CONNABProLie } \subset \operatorname{Kor}(\text { CONNPROLie }) \subseteq \overline{\mathbf{S}}(\text { CONNABProLie })
$$

A discrete abelian group belongs to KOR(CONNPROLIE) precisely if it is finitely generated (and thus lies in CGAL), see 6.6.

\footnotetext{
${ }^{2}$ The discussion of $\operatorname{Aut}\left(\mathfrak{L}\left(\mathrm{SO}_{3} \mathbb{R}\right)\right)$ in [22] p. 252] contains an error; indeed $\mathrm{O}_{3} \mathbb{R} \backslash \mathrm{SO}_{3} \mathbb{R} \nsubseteq \operatorname{Aut}\left(\mathfrak{L}\left(\mathrm{SO}_{3} \mathbb{R}\right)\right)$.
} 


\subsection{Problems.}

(a) Is KOR(CONNPROLIE) closed under $\widehat{\mathbf{Q}}$ ?

(b) Is KOR(CONNPROLIE) closed under $\overline{\mathbf{S}}$ ?

Comments on 9.3. We know that KOR(CONNPROLIE) is not closed under $\mathbf{Q}$ because the group $\mathbb{R}^{\mathbb{R}}$ belongs to KOR(CONNPROLIE) but has a quotient which is not complete (cf. [23, 4.11]), and thus does not lie in KOR(CONNPROLIE) $\subseteq$ ABProLIE.

From 3.6 we know that KOR (CONNPROLIE) is closed under quotients modulo locally compact groups.

9.4 Problem. What about $\operatorname{Kor}(G)$ if $G$ is an almost connected pro-Lie group, or an almost connected locally compact group?

Comments on 9.4. The conclusion of 3.4 breaks down if we drop the assumption of connectedness, cf. 5.2. In many questions about the structure of pro-Lie groups it is possible to weaken a connectedness hypothesis to "almost connected" (i.e., compactness of $\left.G / G_{0}\right)$. For instance, almost connected locally compact groups are pro-Lie groups while for arbitrary disconnected locally groups the homomorphisms into Lie groups need not separate the points. We have seen in 4.8 that $\operatorname{KOR}(\mathrm{ALMCONNLIE})=\operatorname{KOR}(\mathrm{CONNLIE})$ is very well behaved.

The examples in 5.2 fail to be almost connected, and also fail to be pro-Lie groups. The same applies to most of our examples of groups $G \in \mathrm{LCG}$ with $\operatorname{KOR}(G)=G$. Note that the discrete examples are in LIE $\subset$ PROLIE.

9.5 Problems. For $\mathcal{G} \in\{$ LCG, PROLIE $\}$ we ask:

(a) Is CA completely contained in $\operatorname{KOR}(\mathcal{G})$ ?

(b) Is LCA completely contained in $\operatorname{KOR}(\mathcal{G})$ ?

(c) Which part of $\mathrm{CG}$ is contained in $\operatorname{KOR}(\mathcal{G})$ ?

(d) Which discrete groups are in $\operatorname{KOR}(\mathcal{G})$ ?

(e) What is $\operatorname{KOR}(\mathcal{G})$ ?

Comments on 9.5. If we drop all connectedness assumptions on $G$ we obtain examples $G$ where $\operatorname{KOR}(G)$ is not abelian. However, the inclusion $\operatorname{KOR}(\mathcal{G}) \subset \mathcal{G}$ falls far from being an equality. The class $\operatorname{KOR}(\mathcal{G})$ appears to be complicated and not accessible to an easy "constructive" description (such as: "take the following basic examples and use certain constructions like products or quotients").

Definitely, the class CG is not completely contained in $\operatorname{KOR}(\mathcal{G})$. For instance, we know that $\mathrm{CG} \cap \operatorname{KOR}(\mathcal{G})$ is not closed under $\overline{\mathbf{S}}$ or $\mathbf{Q}$, see 8.3 ,

It is also open whether arbitrary discrete groups are in KOR(PROLIE).

\section{Appendix: linear groups}

We collect some known facts regarding the question whether a given group is linear, i.e., admits a faithful ordinary representation. In our present terminology, this means that the trivial group is a member of $\{\operatorname{ker} \rho \mid \rho \in \mathrm{OR}(G)\}$. Examples like the additive group $\mathbb{Z}_{p}$ of $p$-adic integers or any infinite elementary abelian group show that this condition is, in general, much stronger than the condition that $\operatorname{KOR}(G)$ is trivial. 
By way of contraposition, we use the criteria for linearity in certain examples in order to determine $\operatorname{KOR}(G)$ or to even show $\operatorname{KOR}(G)=G$. See 10.8 but also 4.2, 4.3, 5.7,

10.1 Theorem (Mal'tsev [33], see [13]). Let $G$ be a connected Lie group.

(a) The group $G$ is linear if, and only if, its solvable radical and its maximal semisimple subgroups are linear.

(b) If $G$ is semisimple and linear and $Z$ is a discrete normal (i.e., central) subgroup of $G$ then $G / Z$ is linear, as well.

(c) If $G$ is solvable then it is linear if, and only if, it is the semidirect product of a maximal compact subgroup and a simply connected normal subgroup.

10.2 Theorem (Nahlus [36]). Let $G$ be a connected Lie group with Lie algebra $\mathfrak{g}:=\mathfrak{L}(G)$, let $\mathrm{r}$ be the solvable radical of the commutator algebra $\mathrm{g}^{\prime}$, and let $\mathrm{z}$ be the center of $\mathrm{g}$. Choose a maximal torus $T$ of the solvable radical of $G$ and a maximal semisimple subgroup $S$. Then $G$ is linear precisely if $\mathfrak{x} \cap \mathfrak{z} \cap \mathfrak{L}(T)=\{0\}$ and $S$ is linear.

We have seen that the structure of $\operatorname{KOR}(G)$ for a connected Lie group $G$ may depend essentially on the choice of $G$ as a quotient of its simply connected covering $\tilde{G}$. This raises the problem of characterizing those Lie algebras whose associated Lie groups are all linear.

10.3 Theorem (Moskowitz [35]). Let $\mathfrak{g}$ be a Lie algebra of finite dimension over $\mathbb{R}$, let $\mathfrak{r}$ be the solvable radical of the commutator algebra $\mathfrak{g}^{\prime}$, let $\mathrm{z}$ be the center of $\mathfrak{g}$, and let $\mathfrak{s}$ be a maximal semisimple subalgebra of $\mathfrak{g}$. Then every connected Lie group with Lie algebra $\mathfrak{g}$ is linear precisely if the simply connected group associated to $\mathfrak{s}$ is linear and $\mathfrak{r} \cap z=\{0\}$.

10.4 Examples. Among the connected Lie groups with simple Lie algebra, the most obvious non-linear examples are those with infinite center. These are precisely those connected Lie groups with simple Lie algebra where the maximal compact subgroups have centralizers of positive dimension. Cf. [14, Ch. VIII, $\S 6$; Ch. X, $\S 6$ ].

10.5 Theorem (Lee and $\mathrm{Wu}$ [31]). Assume that $\mathrm{G}$ is a connected Lie group, and that $\mathrm{G}$ is linear. Then the holomorph $\operatorname{Aut}(G) \ltimes G$ is linear if, and only if, one of the following holds:

(a) The nilradical of $G$ is simply connected.

(b) The group $G$ is perfect (i.e., coincides with its commutator subgroup).

(c) The quotient $G / G^{\prime}$ is isomorphic to $\mathbb{R} / \mathbb{Z}$.

10.6 Theorem (Burnside [7], cf. [50, Ch. 2, 2.1, Cor. B and C, pp. $138 \mathrm{f}]$ ).

(a) If $G \leq \mathrm{GL}_{n} \mathbb{C}$ has finite exponent (i.e. if there exists $m \geq 1$ such that $\left\{g^{m} \mid g \in G\right\}$ is trivial) then $G$ is a finite group.

(b) If $H \leq \mathrm{GL}_{n} \mathrm{C}$ contains only finitely many conjugacy classes then $H$ is finite.

10.7 Theorem (Schur [43]). If $G \leq \mathrm{GL}_{n} \mathbb{C}$ is a torsion group (i.e., if every element of $G$ has finite order) then every finitely generated subgroup of $G$ is finite.

10.8 Examples. Using Burnside's results we provide examples of (discrete) simple nonabelian groups in KOR(LIE) or even in KOR(SEPLIE) - separability just means countability here. 
(a) For each infinite field $F$ of positive characteristic $p$ the simple group $\mathrm{PSL}_{2} F$ does not admit any non-trivial ordinary representation because it contains an infinite group of exponent $p$, cf. 10.ब(a) Thus $\mathrm{KOR}\left(\mathrm{PSL}_{2} F\right)=\mathrm{PSL}_{2} F$.

(b) There exists a countably infinite group $H$ such that every non-trivial element of $H$ has infinite order, and all these elements form a single conjugacy class, see [16]. Clearly this group $H$ is simple, and $\operatorname{KOR}(H)=H$ follows from 10.6(b).

(c) If $p$ is a sufficiently large prime then there exists a countably infinite group $\mathrm{Olm}_{p}$ such that every proper subgroup of $\mathrm{Olm}_{p}$ has order $p$ and any such subgroup contains a set of representatives for the conjugacy classes, see [37, §19]. We may conclude $\operatorname{KOR}\left(\mathrm{Olm}_{p}\right)=\mathrm{Olm}_{p}$ from any one of Burnside's results as stated in 10.6 , and also from 10.7

\section{References}

[1] I. D. Ado, The representation of Lie algebras by matrices, Uspehi Matem. Nauk (N.S.) 2 (1947), no. 6(22), 159-173, ISSN 0042-1316. MR 0027753 (10,350c)

[2] I. D. Ado, The representation of Lie algebras by matrices, Amer. Math. Soc. Translation 2 (1949), 21, ISSN 0065-9290. MR $0030946(11,77 c)$

[3] S. Ardanza-Trevijano, M. J. Chasco, and X. Domínguez, The role of real characters in the Pontryagin duality of topological abelian groups, J. Lie Theory 18 (2008), no. 1, 193-203, ISSN 0949-5932, http://www.heldermann-verlag.de/jlt/jlt18/chascola2e.pdf. MR 2413959 (2009b:22003) Zbl 1151.22003

[4] R. Bödi and M. Joswig, RealLIE. A software package for real representations of quasi-simple Lie groups, http://www.mathematik.tu-darmstadt.de/ joswig/RealLie/.

[5] R. Bödi and M. Joswig, Tables for an effective enumeration of real representations of quasi-simple Lie groups, Sem. Sophus Lie 3 (1993), no. 2, 239-253, ISSN 0940-2268, http://wWw.heldermann-verlag.de/jlt/jlt03/BOEDIPL.PDF. MR 1270178 (95f:22003) Zbl 0796.22008

[6] N. Bourbaki, General topology. Chapters 1-4, Elements of Mathematics (Berlin), Springer-Verlag, Berlin, 1989, ISBN 3-540-19374-X. MR979294 (90a:54001a) Zbl 0894.54001

[7] W. Burnside, On criteria for the finiteness of the order of a group of linear substitutions, Proc. London Math. Soc. (2) 3 (1905), 435-440, doi:10.1112/plms/s2-3.1.435. JfM 36.0199.02

[8] M. J. Collins, Representations and characters of finite groups, Cambridge Studies in Advanced Mathematics 22, Cambridge University Press, Cambridge, 1990, ISBN 0-521-23440-9. MR 1050762 (91f:20001) Zbl 0703.20001 
[9] J. E. Diem and F. B. Wright, Real characters and the radical of an abelian group, Trans. Amer. Math. Soc. 129 (1967), 517-529, ISSN 0002-9947, http://www.jstor.org/stable/1994605. MR 0214697 (35 \#5546) Zbl 0166.29602

[10] J. Dieudonné, On the automorphisms of the classical groups, Memoirs of the American Mathematical Society 2, American Mathematical Society, Providence, R.I., 1980, ISBN 0-8218-1202-5. MR 606555 (82c:20079) Zbl 0042.25603

[11] P. R. Halmos and H. Samelson, On monothetic groups, Proc. Nat. Acad. Sci. U. S. A. 28 (1942), 254-258, ISSN 0027-8424, http://wwW.pnas.org/content/28/6/254.full.pdf. MR0006543 (4,2f) Zbl 0063.01893

[12] Harish-Chandra, Faithful representations of Lie algebras, Ann. of Math (2) 50 (1949), 68-76, ISSN 0003-486X, doi:10.2307/1969352. MR0028829 (10,504a) Zbl 0032.25201

[13] Harish-Chandra, On faithful representations of Lie groups, Proc. Amer. Math. Soc. 1 (1950), 205-210, ISSN 0002-9939, http: //www. jstor.org/stable/2031923. MR 0034396 (11,579h) Zbl 0039.02004

[14] S. Helgason, Differential geometry, Lie groups, and symmetric spaces, Pure and Applied Mathematics 80, Academic Press Inc., New York, 1978, ISBN 0-12-338460-5. MR514561 (80k:53081) Zbl 0993.53002

[15] E. Hewitt and K. A. Ross, Abstract harmonic analysis. Vol. I, Grundlehren der Mathematischen Wissenschaften 115, Springer-Verlag, Berlin, 2nd edn., 1979, ISBN 3540-09434-2. MR551496 (81k:43001) Zbl 0837.43002

[16] G. Higman, B. H. Neumann, and H. Neumann, Embedding theorems for groups, J. London Math. Soc. 24 (1949), 247-254, ISSN 0024-6107, doi: 10.1112/jlms/s1-24.4.247. MR 0032641 (11,322d) Zbl 0034.30101

[17] K. H. Hofmann, Analytic groups without analysis, in Symposia Mathematica, Vol. XVI (Convegno sui Gruppi Topologici e Gruppi di Lie, INDAM, Rome, 1974), pp. 357-374, Academic Press, London, 1975. MR 0409722 (53 \#13474) Zbl 0319.22021

[18] K. H. Hofmann and S. A. Morris, Free compact groups. I. Free compact abelian groups, Topology Appl. 23 (1986), no. 1, 41-64, ISSN 0166-8641, doi : 10. 1016/0166-8641(86)90016-7. MR 849093 (88a:22011) Zbl 0589.22003

[19] K. H. Hofmann and S. A. Morris, Correction: "Free compact groups. I. Free compact abelian groups" [Topology Appl. 23 (1986), no. 1, 41-64; MR0849093 (88a:22011)], Topology Appl. 28 (1988), no. 1, 101-102, ISSN 0166-8641, doi: 10.1016/0166-8641(88)90040-5. MR 927286 (89a:22008)

[20] K. H. Hofmann and S. A. Morris, Weight and c, J. Pure Appl. Algebra 68 (1990), no. 1-2, 181-194, ISSN 0022-4049, doi:10.1016/0022-4049(90)90142-5. MR 1082789 (92g:22011) Zbl 0728.22006 
[21] K. H. Hofmann and S. A. Morris, The structure of abelian pro-Lie groups, Math. Z. 248 (2004), no. 4, 867-891, ISSN 0025-5874, doi:10.1007/s00209-004-0685-5. MR 2103546 (2005i:22007) Zbl 1058.22003

[22] K. H. Hofmann and S. A. Morris, The structure of compact groups, de Gruyter Studies in Mathematics 25, Walter de Gruyter \& Co., Berlin, augmented edn., 2006, ISBN 978-3-11-019006-9; 3-11-019006-0. MR 2261490 (2007d:22002) Zbl 1139.22001

[23] K. H. Hofmann and S. A. Morris, The Lie theory of connected pro-Lie groups, EMS Tracts in Mathematics 2, European Mathematical Society (EMS), Zürich, 2007, ISBN 978-3-03719-032-6. MR 2337107 (2008h:22001) Zbl 1153.22006

[24] K. H. Hofmann, S. A. Morris, and M. Stroppel, Locally compact groups, residual Lie groups, and varieties generated by Lie groups, Topology Appl. 71 (1996), no. 1, 63-91, ISSN 0166-8641, doi : 10.1016/0166-8641(95)00068-2. MR 1391958 (97k:22006) Zbl 0858.22005

[25] K. H. Hofmann and K.-H. Neeb, Pro-Lie groups which are infinite-dimensional Lie groups, Math. Proc. Cambridge Philos. Soc. 146 (2009), no. 2, 351-378, ISSN 0305-0041, doi:10.1017/S030500410800128X. MR2475971 (2009k:22028) Zbl 1165.22017

[26] K. H. Hofmann and K.-H. Neeb, The compact generation of closed subgroups of locally compact groups, J. Group Theory 12 (2009), no. 4, 555-559, ISSN 1433-5883, doi:10.1515/JGT.2008.096. MR 2542208 Zbl 1179.22003

[27] K. Iwasawa, On the representation of Lie algebras, Jap. J. Math. 19 (1948), 405-426. MR 0032613 (11,317d)

[28] K. Iwasawa, On some types of topological groups, Ann. of Math. (2) 50 (1949), 507-558, ISSN 0003-486X, doi: 10.2307/1969548. MR 0029911 (10,679a) Zbl 0034.01803

[29] I. Kaplansky, Lie algebras and locally compact groups, The University of Chicago Press, Chicago, Ill.-London, 1971. MR 0276398 (43 \#2145) Zbl 0223.17001

[30] S. Lang, Algebra, Addison-Wesley Publishing Company Advanced Book Program, Reading, MA, 2nd edn., 1984, ISBN 0-201-05487-6. MR 783636 (86j:00003) Zbl 0848.13001

[31] D. H. Lee and T. S. Wu, On faithful representations of the holomorph of Lie groups, Math. Ann. 275 (1986), no. 3, 521-527, ISSN 0025-5831, doi: 10.1007/BF01458621. MR 858294 (87k:22012) Zbl 0603.22002

[32] G. W. Mackey, On induced representations of groups, Amer. J. Math. 73 (1951), 576-592, ISSN 0002-9327, doi:10.2307/2372309. MR0042420 (13,106d) Zbl 0045.30305

[33] A. I. Mal'tsev, On linear Lie groups, C. R. (Doklady) Acad. Sci. URSS (N. S.) 40 (1943), 87-89. MR $0013164(7,115 b)$ Zbl 0061.04704 
[34] D. Montgomery and L. Zippin, Topological transformation groups, Interscience Publishers, New York-London, 1955. MR 0073104 (17,383b) Zbl 0068.01904

[35] M. Moskowitz, A remark on faithful representations, Atti Accad. Naz. Lincei Rend. Cl. Sci. Fis. Mat. Natur. (8) 52 (1972), 829-831 (1973). MR0327979 (48 \#6321) Zbl 0294.22011

[36] N. Nahlus, Note on faithful representations and a local property of Lie groups, Proc. Amer. Math. Soc. 125 (1997), no. 9, 2767-2769, ISSN 0002-9939, doi : 10. 1090/S0002-9939-97-03893-8. MR 1396990 (97j:22018) Zbl 0882.22009

[37] A. Y. Ol'shanski1̌, Geometry of defining relations in groups, Mathematics and its Applications (Soviet Series) 70, Kluwer Academic Publishers Group, Dordrecht, 1991, ISBN 0-7923-1394-1. MR 1191619 (93g:20071) Zbl 0732.20019

[38] A. L. Onishchik and È. B. Vinberg, Lie groups and algebraic groups, Springer Series in Soviet Mathematics, Springer-Verlag, Berlin, 1990, ISBN 3-540-50614-4. MR 1064110 (91g:22001) Zbl 0722.22004

[39] D. J. S. Robinson, A course in the theory of groups, Graduate Texts in Mathematics 80, Springer-Verlag, New York, 2nd edn., 1996, ISBN 0-387-94461-3. MR 1357169 (96f:20001) Zbl 0836.20001

[40] W. Roelcke and S. Dierolf, Uniform structures on topological groups and their quotients, McGraw-Hill International Book Co., New York, 1981, ISBN 0-07-0543412-8. MR 644485 (83j:22001) Zbl 0489.22001

[41] H. Salzmann, D. Betten, T. Grundhöfer, H. Hähl, R. Löwen, and M. Stroppel, Compact projective planes, de Gruyter Expositions in Mathematics 21, Walter de Gruyter \& Co., Berlin, 1995, ISBN 3-11-011480-1. MR 1384300 (97b:51009) Zbl 0851.51003

[42] O. Schreier and B. L. van der Waerden, Die Automorphismen der projektiven Gruppen, Abh. Math. Sem. Univ. Hamburg 6 (1928), 303-322, doi : 10. 1007/BF02940620. JfM 54.0149.02

[43] I. Schur, Über Gruppen periodischer linearer Substitutionen., Sitzungsber. Preuss. Akad. Wiss. (1911), 619-627. JfM 42.0155.01

[44] M. Stroppel, Locally compact groups with many automorphisms, J. Group Theory 4 (2001), no. 4, 427-455, ISSN 1433-5883, doi:10.1515/jgth.2001.032. MR 1859180 (2002g:22010) Zbl 0999.22006

[45] M. Stroppel, Locally compact groups, EMS Textbooks in Mathematics, European Mathematical Society (EMS), Zürich, 2006, ISBN 3-03719-016-7, doi : 10.4171/016. MR 2226087 (2007d:22001) Zbl 1102.22005

[46] W. T. van Est, On Ado's theorem, Nederl. Akad. Wetensch. Proc. Ser. A $69=$ Indag. Math. 28 (1966), 176-191. MR 0201576 (34 \#1458) Zbl 0156.03901 
[47] B. A. F. Wehrfritz, Infinite linear groups. An account of the group-theoretic properties of infinite groups of matrices, Springer-Verlag, New York, 1973. MR 0335656 (49 \#436) Zbl 0261.20038

[48] F. B. Wright, Topological abelian groups, Amer. J. Math. 79 (1957), 477-496, ISSN 00029327, doi : 10.2307/2372559. MR0089361 (19,662a) Zbl 0079.25604

[49] H. Yamabe, A generalization of a theorem of Gleason, Ann. of Math. (2) 58 (1953), no. 2, 351-365, ISSN 0003-486X, doi:10.2307/1969792. MR0058607 (15,398d) Zbl 0053.01602

[50] A. E. Zalesskiŭ, Linear groups, in Current problems in mathematics. Fundamental directions, Vol. 37 (Russian), Itogi Nauki i Tekhniki, pp. 114-228, 236, Akad. Nauk SSSR Vsesoyuz. Inst. Nauchn. i Tekhn. Inform., Moscow, 1989. MR 1039816 (91m:20003) Zbl 0715.20027

Acknowledgement. A substantial part of these notes was written while the author was a guest of SFB 478 "Geometrische Strukturen in der Mathematik", Münster, Germany.

\section{Author's address:}

Markus Stroppel, Fachbereich Mathematik, Universität Stuttgart, D-70550 Stuttgart, Germany. 